\title{
Comparative genome characterization of the periodontal pathogen Tannerella forsythia
}

Nikolaus F. Zwickl', Nancy Stralis-Pavese ${ }^{1}$, Christina Schäffer ${ }^{2}$, Juliane C. Dohm ${ }^{1 *}$ and Heinz Himmelbauer ${ }^{1 *}$ (D)

\begin{abstract}
Background: Tannerella forsythia is a bacterial pathogen implicated in periodontal disease. Numerous virulenceassociated T. forsythia genes have been described, however, it is necessary to expand the knowledge on $T$. forsythia's genome structure and genetic repertoire to further elucidate its role within pathogenesis. Tannerella sp. BU063, a putative periodontal health-associated sister taxon and closest known relative to T. forsythia is available for comparative analyses. In the past, strain confusion involving the T. forsythia reference type strain ATCC 43037 led to discrepancies between results obtained from in silico analyses and wet-lab experimentation.

Results: We generated a substantially improved genome assembly of T. forsythia ATCC 43037 covering 99\% of the genome in three sequences. Using annotated genomes of ten Tannerella strains we established a soft core genome encompassing 2108 genes, based on orthologs present in $>=80 \%$ of the strains analysed. We used a set of known and hypothetical virulence factors for comparisons in pathogenic strains and the putative periodontal healthassociated isolate Tannerella sp. BU063 to identify candidate genes promoting T. forsythia's pathogenesis. Searching for pathogenicity islands we detected 38 candidate regions in the T. forsythia genome. Only four of these regions corresponded to previously described pathogenicity islands. While the general protein O-glycosylation gene cluster of T. forsythia ATCC 43037 has been described previously, genes required for the initiation of glycan synthesis are yet to be discovered. We found six putative glycosylation loci which were only partially conserved in other bacteria. Lastly, we performed a comparative analysis of translational bias in T. forsythia and Tannerella sp. BU063 and detected highly biased genes.

Conclusions: We provide resources and important information on the genomes of Tannerella strains. Comparative analyses enabled us to assess the suitability of $T$. forsythia virulence factors as therapeutic targets and to suggest novel putative virulence factors. Further, we report on gene loci that should be addressed in the context of elucidating T. forsythia's protein O-glycosylation pathway. In summary, our work paves the way for further molecular dissection of $T$. forsythia biology in general and virulence of this species in particular.
\end{abstract}

Keywords: Tannerella, Genome assembly, Comparative genomics, Pan-genome, Virulence, Pathogenicity island, Glycosylation gene cluster, Codon usage bias, Computational analysis, Periodontitis

\footnotetext{
* Correspondence: dohm@boku.ac.at; heinz.himmelbauer@boku.ac.at

'Department of Biotechnology, Institute of Computational Biology, University

of Natural Resources and Life Sciences (BOKU), Vienna, Austria

Full list of author information is available at the end of the article
}

(c) The Author(s). 2020 Open Access This article is distributed under the terms of the Creative Commons Attribution 4.0 International License (http://creativecommons.org/licenses/by/4.0/), which permits unrestricted use, distribution, and reproduction in any medium, provided you give appropriate credit to the original author(s) and the source, provide a link to the Creative Commons license, and indicate if changes were made. The Creative Commons Public Domain Dedication waiver (http://creativecommons.org/publicdomain/zero/1.0/) applies to the data made available in this article, unless otherwise stated. 


\section{Background}

Tannerella forsythia is a bacterial pathogen associated with human periodontitis, a polymicrobial inflammatory disease of tooth-surrounding tissues [1]. Numerous genes of $T$. forsythia have been reported in the context of the pathogenesis of the disease. Examples include welldescribed virulence factors such as the leucine-rich-repeat protein BspA [2, 3] and the protease PrtH/Fdf [4]. The T. forsythia cell surface (S-) layer was described to consist of the alternating TfsA and TfsB glycoproteins that have their corresponding genes located next to each other in the genome [5-7] and align in a 2D lattice, which drastically impacts the host immune response [8-10]. In $T$. forsythia, the S-layer proteins as well as other cell surface proteins are modified with a complex $O$-glycan that can be dissected in a species-specific portion and a core saccharide that is proposed to be conserved in the Bacteroidetes phylum of bacteria $[6,10,11]$. A multi-gene locus encoding the species-specific part of the T. forsythia protein $O$-glycan was identified, and the corresponding protein $O$-glycosylation pathway has been recently explored in detail [10]. Following assembly of the glycoprotein in the bacterial periplasm, the S-layer glycoproteins are targeted via their conserved C-terminal domain (CTD) to a type IX secretion system (T9SS) for export across the outer membrane [12]. The T9SS is a recently discovered, complex translocon found only in some species of the Bacteroidetes phylum [13], and CTDs, typically consisting of 40-70 amino acids and sharing an immunoglobulinsuperfamily (IgSF) domain, are present in many other proteins in $T$. forsythia. The glycobiology repertoire of the $T$. forsythia genome also contains numerous glycosidases and carbohydrate-active enzymes that require attention within the context of virulence [14]. Further, a sialic acid utilization gene locus encoding a transporter and involved enzymes have been shown to play an important role for the species to thrive within the oral biofilm community [15-17]. Apart from the capability of cleaving oligosaccharides, the niche and suggested role in pathogenesis requires the species to produce proteolytic enzymes; in addition to PrtH, much attention has been directed to a set of six proteases of similar protein architecture which contain a modified CTD, terminating with the amino acid sequence KLIKK, hence termed KLIKK proteases [18]. Whereas the roles of these and other suggested virulence factors continue to be explored, the search for novel virulence factors may be required to complete the picture on T. forsythia's contributions and role in pathogenesis.

Previous characterizations of the $T$. forsythia virulence factors were mostly based on the American Type Culture Collection (ATCC) 43037 type strain employing wet-lab experimentation, whereas computational analyses of the virulence-related gene repertoire mostly used the genome sequence of strain FDC 92A2. Although FDC 92A2 was the first fully sequenced $T$. forsythia strain available [19], the resulting genome assembly was incorrectly labelled and deposited as ATCC 43037 in the National Center for Biotechnology Information (NCBI) databases. This discrepancy was not noticed by the research community until many years later. Because of inconsistent results and sequence mismatches, initially interpreted as sequencing errors or as misassemblies in the genomic reference, $T$. forsythia was sequenced again and a genuine genome assembly for ATCC 43037 was generated [20]. Meanwhile, the strain attribution error has been corrected in the NCBI databases, but persists in other databases.

The T. forsythia ATCC 43037 genome assembly published by Friedrich et al. was a draft genome assembly, consisting of 141 contigs with an N50 contig length of 110 kbp. Even though this has substantially improved the genomics resources available for $T$. forsythia, a more contiguous and more complete genome assembly is required for many analyses, especially for whole-genome comparative approaches. Furthermore, the genome assembly of strain FDC 92A2 remained in the NCBI databases as reference genome for $T$. forsythia due to its completeness. However, the cultivation of FDC 92A2 has been reported to be unreliable [21], so that ATCC 43037 will certainly continue to be the most widely used strain in research labs. In addition to the genome assemblies of ATCC 43037 and FDC 92A2, genome assemblies of eight further $T$. forsythia strains have become available in recent years [22-25].

Within the genus Tannerella, T. forsythia is the only well characterized species. Several isolates from various origins have been assigned to the genus Tannerella [26]; until recently, however, none of these have been successfully cultivated, hampering their characterization.

Tannerella sp. BU063 (also referred to as Human Microbial Taxon ID 286 or HMT 286) is of special interest, as it is considered a putative periodontal health-associated strain. Following recent successful cultivation [27], a complete and gap-free genome assembly of Tannerella sp. BU063 has become available replacing a previously generated highly fragmented assembly [28].

Overall, the currently available genomes from the genus Tannerella enable comparative genomics approaches to (i) continue searching for novel $T$. forsythia virulence factors, (ii) confirm the relevance of previously reported or suggested virulence factors throughout the $T$. forsythia species, and (iii) explore features of the $T$. forsythia genome that might be of interest beyond the organism's virulence.

Here, we present a new, more contiguous genome assembly for the T. forsythia ATCC 43037 type strain, which is based on sequences of the published draft assembly and, hence, is compatible with previous studies and gene annotations. Further, we use this improved genome assembly together with genome assemblies from nine additional $T$. forsythia isolates and from the putative health-associated 
relative Tannerella sp. BU063 in comparative genomics approaches.

\section{Results}

Improved assembly of the Tannerella forsythia type strain ATCC 43037

The genome of the T. forsythia ATCC 43037 type strain had been assembled previously [20] based on Illumina paired-end sequencing data resulting in an assembly of 141 contigs with an N50 size of 114 kilobasepairs (kbp) (Table 1). The largest sequence was $487 \mathrm{kbp}$ comprising about $15 \%$ of the total assembly size of 3.282 Megabasepairs (Mbp). In order to improve the contiguity of the assembly, we generated a new data set of 11 million Illumina mate-pairs with read length of $2 \times 125$ nucleotides (nt), corresponding to 800 -fold genome coverage, and showing a peak span size of $1.8 \mathrm{kbp}$ (Additional file 10: Figure S1). We used both the published paired-end sequencing reads downsampled to a coverage of 100-fold and the newly generated mate-pairs to build connections between the contigs of the ATCC 43037 genome assembly generated by Friedrich et al. [20]. After scaffolding and gap filling, the N50 length increased to $1.85 \mathrm{Mbp}$ and the number of sequences decreased to 87 . The total assembly size increased slightly to $3.296 \mathrm{Mbp}$ due to gaps between contigs. The three largest sequences (1.85 Mbp, $859 \mathrm{kbp}$, $532 \mathrm{kbp}$ ) encompassed $99.1 \%$ of the assembly. The fraction of undetermined bases within scaffolds was very small (0.26\%). Thus, the new assembly of strain ATCC 43037 can be considered as essentially complete. The genome sizes of three fully sequenced $T$. forsythia strains were slightly larger, namely 3.40 Mbp (FDC 92A2) [19], 3.39 Mbp (KS16), and $3.35 \mathrm{Mbp}$ (3313) [22], respectively, with an average genome size of $3.38 \mathrm{Mbp}$. Taking this average genome size as a basis the average gap size in the new ATCC 43037 assembly was 900 bp between scaffolds.

We compared our ATCC 43037 assembly to a published 15 kbp-long genomic sequence (GenBank accession KP715369) of the same T. forsythia strain [18] resulting in a conflicting alignment. About one half of the sequence published by Ksiazek et al. aligned to a non-terminal region in scaffold 1 and the other half aligned to a non-terminal region in scaffold 2 in our assembly. We carefully checked the sequencing reads that supported our connections and also mapped our reads to the $15-\mathrm{kbp}$ sequence. Reduced read coverage was found in all breakpoint regions, but several thousands of connecting mate-pairs supported our version compared to only twenty mate-pairs that would confirm the continuity of the 15-kbp sequence (Fig. 1). When comparing the 15-kbp sequence to the published genome assemblies of T. forsythia strains 92A2, 3313, and KS16, we did not find the $15-\mathrm{kb}$ sequence to align continuously in any of these strains, however, the majority of the produced alignments were found within single regions of each of the three genomes. While some parts of the $15-\mathrm{kbp}$ sequence aligned also to other regions, a distinct split, as described above for ATCC 43037, could not be observed (Additional file 12: File S1). We note that Ksiazek et al. published their work at a time when it was not yet clear that the T. forsythia reference genome attributed to ATCC 43037 was in fact derived from strain 92A2 [20]. Hence, Ksiazek et al. may have unknowingly relied on strain 92A2 instead of ATCC 43037 for guiding their sequencing and assembly strategy.

\section{Comparative analysis of Tannerella sp. genome assemblies}

Our new genome sequence allowed whole-genome comparisons with other Tannerella assemblies to assess

Table 1 Tannerella genome assemblies analysed including the ATCC 43037 assembly generated in this work

\begin{tabular}{|c|c|c|c|c|c|}
\hline Strain name & GenBank Accession & Genome size [bp] & \# of sequences & $\%$ GC & RefSeq Annotation Date \\
\hline \multicolumn{6}{|c|}{ Tannerella forsythia } \\
\hline ATCC 43037 & VFJI00000000 (this work) & $3,296,274$ & 87 & 47.1 & - \\
\hline ATCC 43037 & JUET00000000.1 & $3,281,748$ & 141 & 47.1 & $06 / 12 / 2017$ \\
\hline FDC $92 \mathrm{~A} 2$ & NC_016610.1 & $3,405,521$ & 1 & 47.0 & $10 / 21 / 2017$ \\
\hline 3313 & NZ_AP013044.1 & $3,350,939$ & 1 & 47.1 & $04 / 04 / 2017$ \\
\hline KS16 & NZ_AP013045.1 & $3,393,002$ & 1 & 47.2 & $04 / 04 / 2017$ \\
\hline UB4 & FMMN00000000.1 & $3,233,032$ & 71 & 47.2 & $06 / 12 / 2017$ \\
\hline UB22 & FMML00000000.1 & $3,272,368$ & 98 & 47.1 & $06 / 12 / 2017$ \\
\hline UB20 & FMMM00000000.1 & $3,252,894$ & 93 & 47.1 & $06 / 12 / 2017$ \\
\hline 9610 & MEHX00000000.1 & $3,201,941$ & 79 & 47.3 & $06 / 12 / 2017$ \\
\hline W11663 & NSLJ00000000.1 & $3,300,179$ & 140 & 47.1 & $10 / 14 / 2017$ \\
\hline W10960 & NSLK00000000.1 & $3,312,685$ & 98 & 47.2 & $10 / 14 / 2017$ \\
\hline \multicolumn{6}{|c|}{ Tannerella sp. BU063 } \\
\hline $\mathrm{n} / \mathrm{a}$ & CP017038.1 & $2,973,531$ & 1 & 56.5 & $04 / 13 / 2017$ \\
\hline
\end{tabular}




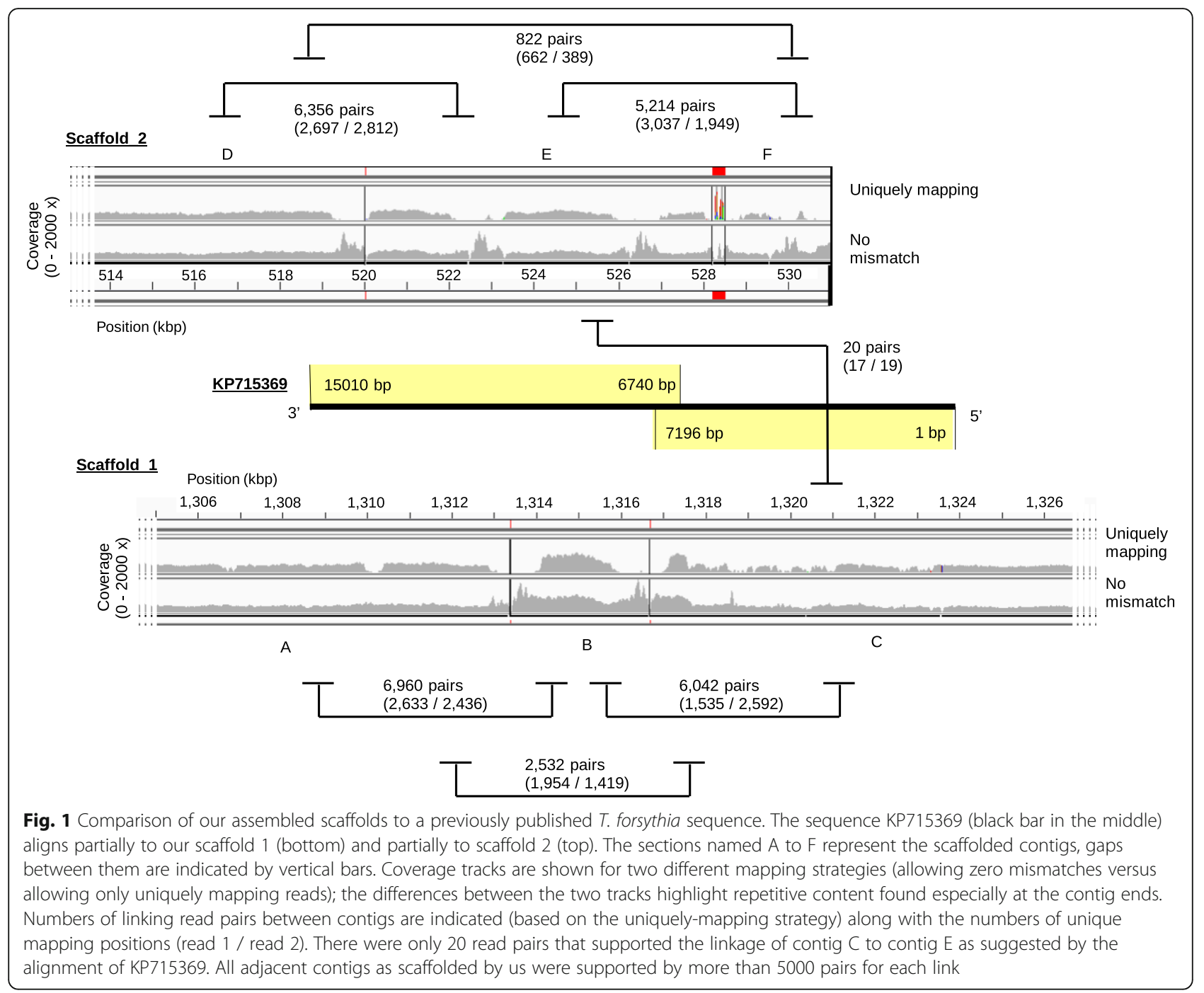

genomic structural differences and gene order conservation. We compared the available genome assemblies of six disease-associated T. forsythia strains - 92A2, 3313, KS16, UB4, UB20, and UB22 - with the assembly of strain ATCC 43037, together with the putative health-associated Tannerella sp. BU063 isolate in whole-genome alignments (Table 1). Genome assemblies of a close relative of Tannerella sp. BU063 dubbed Tannerella sp. BU045 were recently released [29] based on data that were acquired by single-cell sequencing. Considering the degree of assembly fragmentation (about 600 contigs, N50 of about $22 \mathrm{kbp}$ ), data derived from this isolate were not used for the current work. We chose strain $92 \mathrm{~A} 2$ as a reference because of its completeness and aligned the other strains against it. The alignments revealed that all $T$. forsythia strains shared highly conserved genome structures (Fig. 2). Three of the assemblies showed considerable fragmentation (strain UB4: 71 contigs, UB20: 93 contigs, UB22: 98 contigs) so that large-scale rearrangements could not be analysed.
However, $78-83 \%$ of the assembled contigs per strain aligned to strain $92 \mathrm{~A} 2$ with at least $80 \%$ of their length and minimal sequence identity of $80 \%$, taking alignments with a minimum length of $250 \mathrm{bp}$ into account. Only a few contigs that could not be aligned to the 92A2 reference under these conditions exceeded 1000 bp (one, six, and seven contigs for UB4, UB20, and UB22, respectively), comprising only $2-8 \%$ of the total assembly lengths (Table 2). Reducing the required alignment length from 80 to $50 \%$, more than $99.5 \%$ of each assembly aligned to the $92 \mathrm{~A} 2$ reference. Similarity blocks as detected throughout all compared strains spanned contig boundaries in many cases suggesting a high degree of collinearity even between the fragmented assemblies.

The genomes of strains 92A2, 3313 and KS16 had been assembled into one contiguous sequence, and, thus, were most informative regarding potential rearrangements within the T. forsythia species. The alignments confirmed 


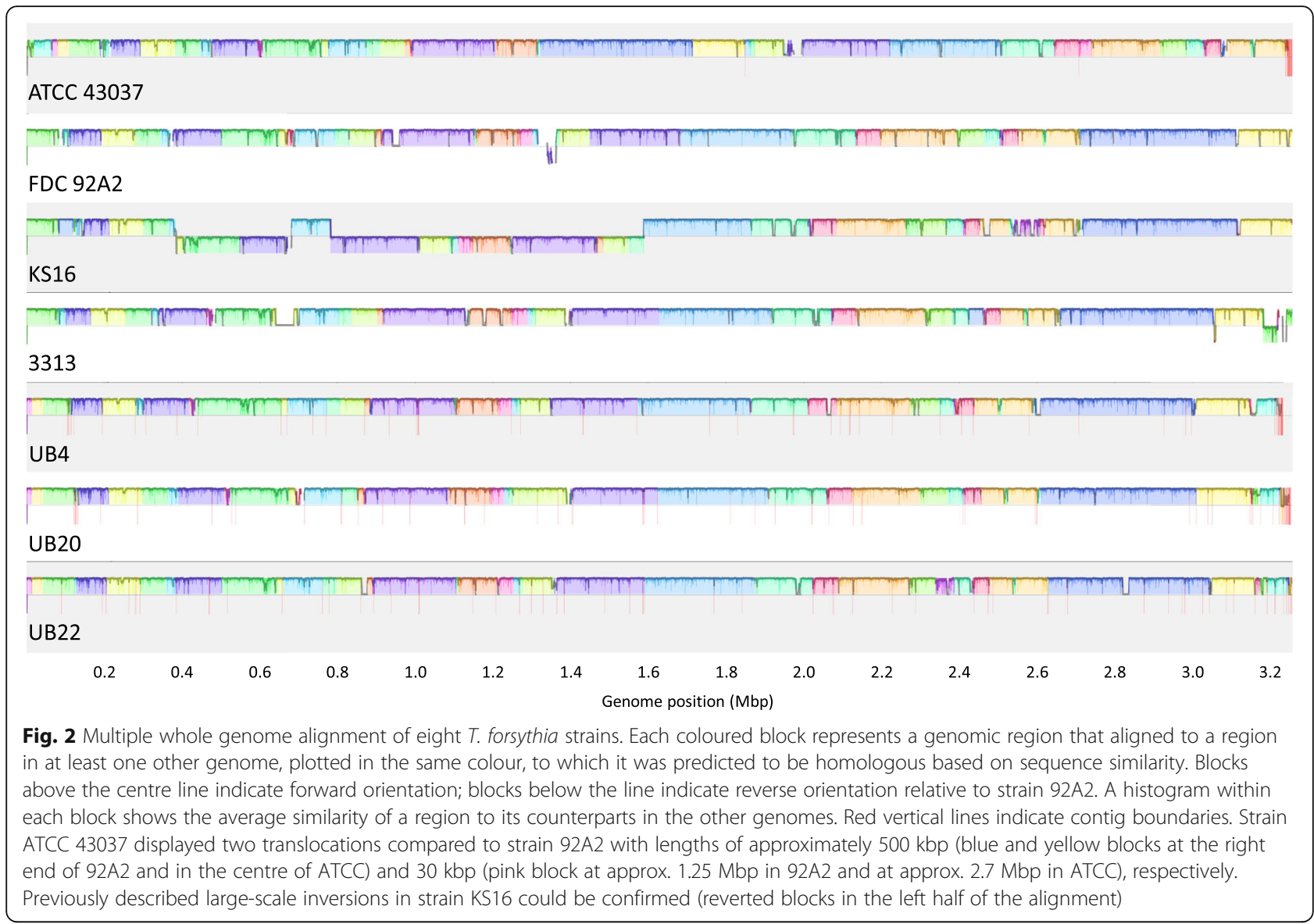

Table 2 Alignable fraction of nine T. forsythia strains and Tannerella sp. BU063 in whole-genome alignments against T. forsythia strain FDC 92A2 as reference sequence. Results are based on blastn output. The scaffolded ATCC 43037 assembly generated in this work was used

\begin{tabular}{|c|c|c|c|c|c|}
\hline \multicolumn{6}{|l|}{ Strain name } \\
\hline & $>=99 \%$ seq identity & $>=95 \%$ seq identity & $>=80 \%$ seq identity & $>=70 \%$ seq identity & $>=50 \%$ seq identity \\
\hline \multicolumn{6}{|c|}{ Tannerella forsythia } \\
\hline ATCC 43037 & 40.58 & 88.52 & 91.46 & 92.15 & 92.59 \\
\hline 3313 & 44.27 & 87.68 & 92.00 & 92.56 & 92.76 \\
\hline KS16 & 43.43 & 90.63 & 92.72 & 93.24 & 93.55 \\
\hline UB4 & 42.61 & 88.47 & 92.59 & 93.14 & 93.29 \\
\hline UB22 & 51.94 & 90.99 & 92.02 & 93.01 & 93.36 \\
\hline UB20 & 49.89 & 90.54 & 93.30 & 93.68 & 93.89 \\
\hline 9610 & 42.58 & 87.86 & 90.35 & 90.87 & 91.21 \\
\hline W11663 & 47.50 & 90.30 & 92.50 & 92.94 & 93.06 \\
\hline W10960 & 44.83 & 88.75 & 91.70 & 92.55 & 92.92 \\
\hline average & 45.29 & 89.30 & 92.07 & 92.68 & 92.96 \\
\hline \multicolumn{6}{|c|}{ Tannerella sp. BU063 } \\
\hline & $>=95 \%$ seq identity & $>=80 \%$ seq identity & $>=70 \%$ seq identity & $>=50 \%$ seq identity & $>=30 \%$ seq identity \\
\hline $\mathrm{n} / \mathrm{a}$ & 0.00 & 0.97 & 24.38 & 38.25 & 38.37 \\
\hline
\end{tabular}


two large inversions in strain KS16 when compared to $92 \mathrm{~A} 2$ or 3313 , and a high degree of collinearity between the latter two, as reported previously [22]. Our ATCC 43037 assembly was found to show two large-scale rearrangements when compared to strains $92 \mathrm{~A} 2$ and 3313, respectively. One of these rearrangements disrupted the larger of the two KLIKK protease loci, which was contained within the 15-kbp sequence mentioned above.

In order to investigate the relatedness among the $10 \mathrm{~T}$. forsythia strains and Tannerella sp. BU063, we performed a phylogenetic analysis. We determined pairwise distances between the assembled genomes using Mash [30] and included Bacteroides vulgatus ATCC 8482 as an outgroup. The resulting distance matrix was used to calculate a phylogentic tree using the Fitch-Margoliash algorithm. The phylogenetic tree clustered the ten T. forsythia isolates closely together and showed Tannerella sp. BU063 as a separate sister taxon. The distance of $T$. sp. BU063 to the T. forsythia subtree was almost as large as the distance of the outgroup (Fig. $3 \mathrm{a}, \mathrm{b}$ ).

We found large differences to the genome structure of the putative periodontal health-associated isolate Tannerella sp. BU063. When aligning the genome assemblies of nine disease-associated strains - ATCC 43037, 3313, KS16, UB4, UB20, UB22, 9610, WW11663, and WW10960 - to the genome of strain $92 \mathrm{~A} 2$, on average $92.1 \%$ of the $92 \mathrm{~A} 2$ sequence was covered (match length cut-off $250 \mathrm{bp}$; minimum sequence identity $80 \%$ ), and 41 to $52 \%$ were found to be covered even when raising the sequence identity threshold to $99 \%$. In contrast, the genome sequences of the putative periodontal health-associated phylotype Tannerella sp. BU063 covered less than $1 \%$ of the $92 \mathrm{~A} 2$ genome by alignments with a sequence identity of at least $80 \%$. Even when lowering the sequence identity cut-off to 70 and $50 \%$ the alignments covered only 24 and $38 \%$ of the $92 \mathrm{~A} 2$ sequence, respectively.

Similarly, our findings confirmed that the gene order in T. forsythia compared to Tannerella sp. BU063 was largely changed. Loss of synteny had been reported previously based on highly fragmented genome assemblies [28]. Here, we used the complete and gap-free genome sequence of Tannerella sp. BU063 (Table 1) enabling genome-wide analysis beyond previous breakpoints. Although $55 \%$ of the genes encoded within the Tannerella sp. BU063 genome were found to have an ortholog in at least six different $T$. forsythia strains, our genomic alignment indicated that the gene order was shuffled (Fig. 4).

In each of the assemblies of 3313, 92A2, and ATCC 43037 we found one continuous sequence of at least 20 $\mathrm{kbp}$ that indicated a strain-specific region to which no other strain contained a homologous segment that could be aligned well. The strains KS16 and 3313, both of them isolated from periodontitis patients in Japan, shared a homologous block that was specific to these two strains which encompassed a gene annotated as a transposase, surrounded by numerous genes that had been annotated as hypothetical proteins of unknown function [22]. We expect further strain-specific regions of similar size as well as strain-specific genes in the other genomes. The individual location of strain-specific regions in 3313, 92A2, ATCC 43037 suggested that such regions occur dispersed throughout the genomes.

In summary, these results and the alignments shown in Fig. 2 illustrate the high degree of conservation with respect to sequence content as well as genome structure throughout the T. forsythia species and provide genomic evidence to suggest the re-classification of Tannerella sp. BU063 as a separate species.

\section{Comparative assessment of Tannerella virulence factors}

Currently available T. forsythia genomes contain 2600 2700 protein-coding genes, many of which lack functional annotation. The increasing wealth of knowledge contained in sequence databases may provide functional predictions for these genes in the future. At present, however, we may reveal candidate genes involved in pathogenesis by comparing complete genomes from strains of known pathogenic and non-pathogenic nature, even if their genes are not yet functionally annotated. Such an approach is especially interesting in the case of T. forsythia, as its cultivation requirements make a systematic knock-out approach very challenging.

A number of genes have so far been suggested to be associated with the pathogenicity of T. forsythia [18, 31-33]. We assessed the presence or absence of functional orthologs of such genes within genome assemblies of ten different $T$. forsythia strains, as well as within the putative periodontal health-associated genome of Tannerella sp. BU063. We employed BLAST score ratio (BSR) values for the gene comparisons as calculated with LS-BSR [34], whereby the blast score of the alignment of two genes that match each other is normalized by dividing the result by the blast score obtainable in a self-hit of the query. This yields a value of 1 for identical sequences and a value of zero for sequences which are entirely unrelated. We included 45 potential virulence-related genes and determined their BSR values in all eleven strains by applying LS-BSR on the entire genomes (Fig. 5, Additional file 1: Table S1) and on the annotated gene sets (Additional file 11: Figure S2, Additional file 2: Table S2). High BSR values suggest that a functional ortholog to a pathogenicity-associated gene is present in a certain strain, while BSR values $<0.4$ indicate likely absence of a functional ortholog of this gene [34]. The two input data sets resulted in comparable BSR values for most genes. Differences in BSR values (differing by 0.2 or more: TfsA in one strain, mirolysin in one strain, karilysin in two strains, and TF2392 in three strains) might indicate incorrectly annotated genes in particular strains or 


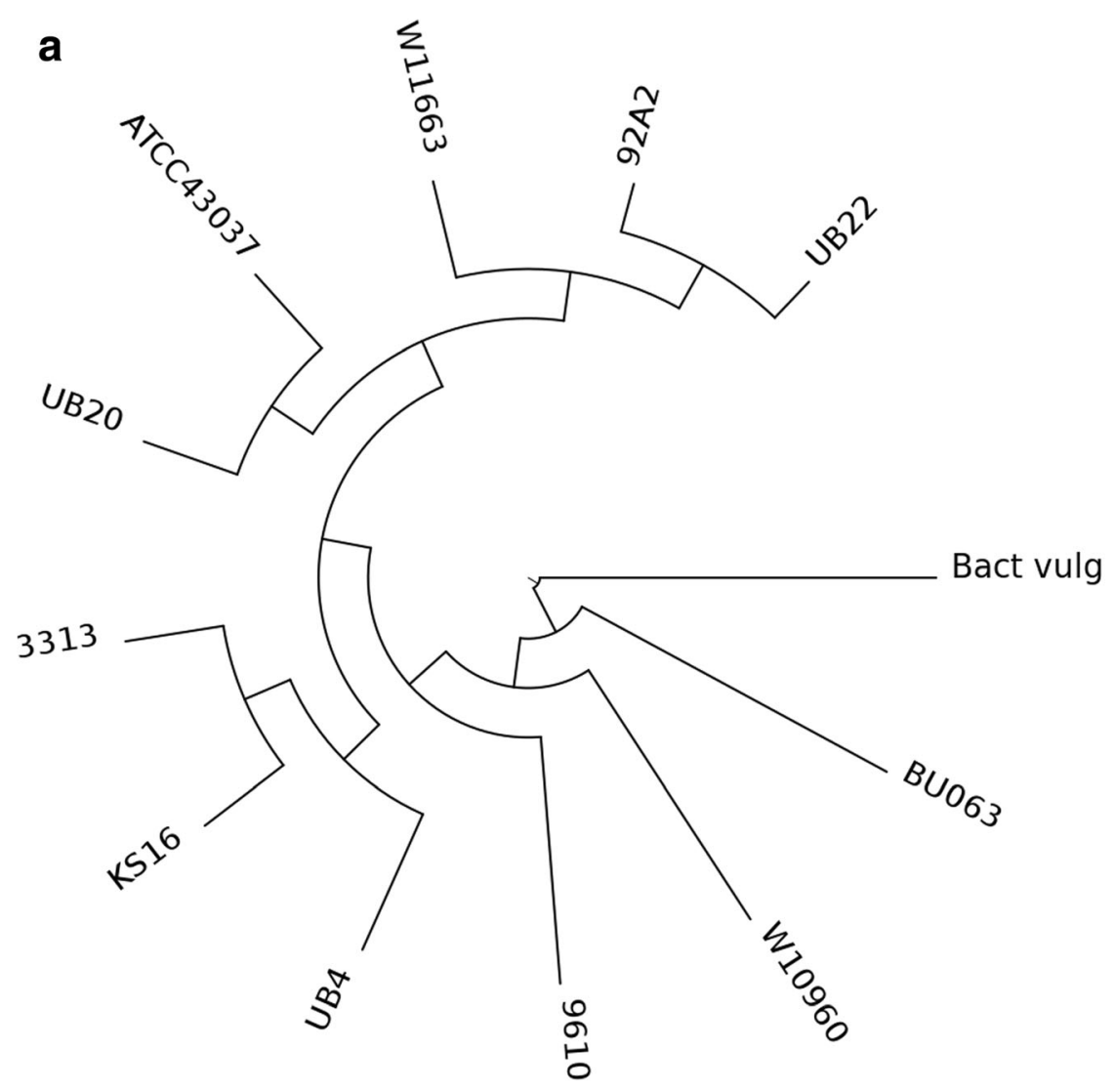

b

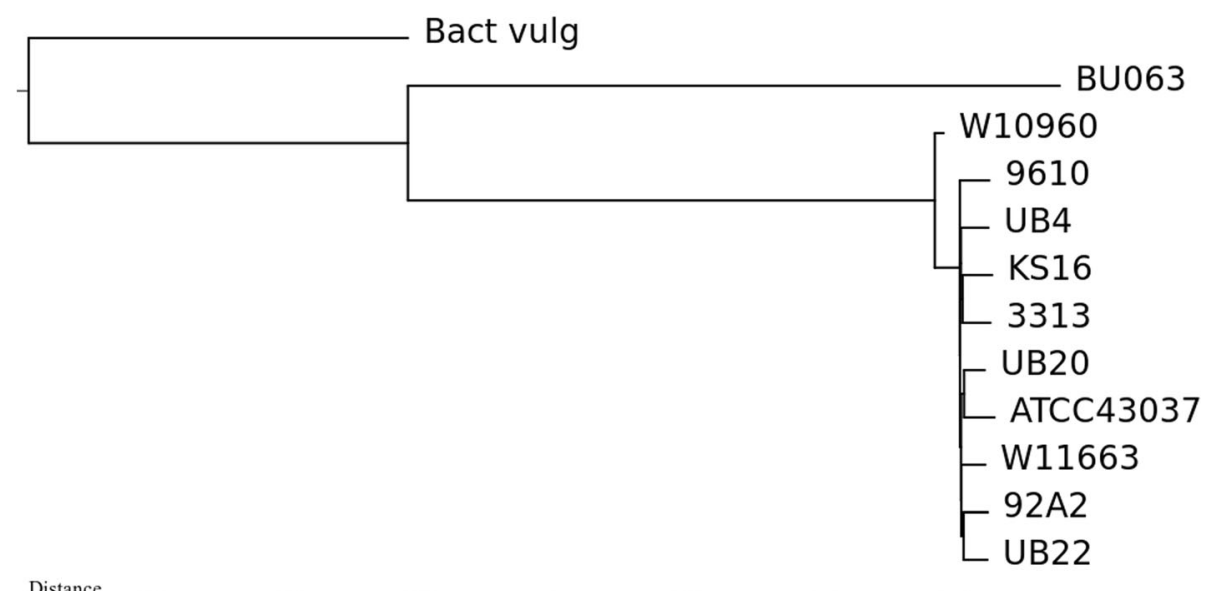

$\begin{array}{lllllll}\text { Distance } & 0.025 & 0.05 & 0.075 & 0.1 & 0.125 & 0.15\end{array}$

Fig. 3 Phylogenetic tree showing the topology (a) and the distances (b) as computed by MASH applied on the whole-genome assemblies of $T$. forsythia strains and Tannerella sp. BU063, including Bacterioides vulgatus ATCC 8482 as outgroup

truncated gene sequences due to mutations of start or stop codons.

Based on the comparison of entire genomes our result showed generally high BSR values for virulence factors in the pathogenic T. forsythia strains and low BSR values in Tannerella sp. BU063 (Fig. 5, Additional file 1: Table S1). However, BSR values > 0.7 indicated 11 pathogenicity- associated genes as present in Tannerella sp. BU063 (of which four genes had BSR > = 0.9: methylglyoxal synthase, GroEL, enolase, TF2925). Four genes with BSR $<0.4$ indicated absence in at least one of the pathogenic strains (forsilysin in strain 9610; BspA_2 in UB20; AbfA in 3313; TF1589 in ATCC 43037, UB4, UB22, and 9610) (Additional file 1: Table S1) providing evidence that re-evaluation of the 


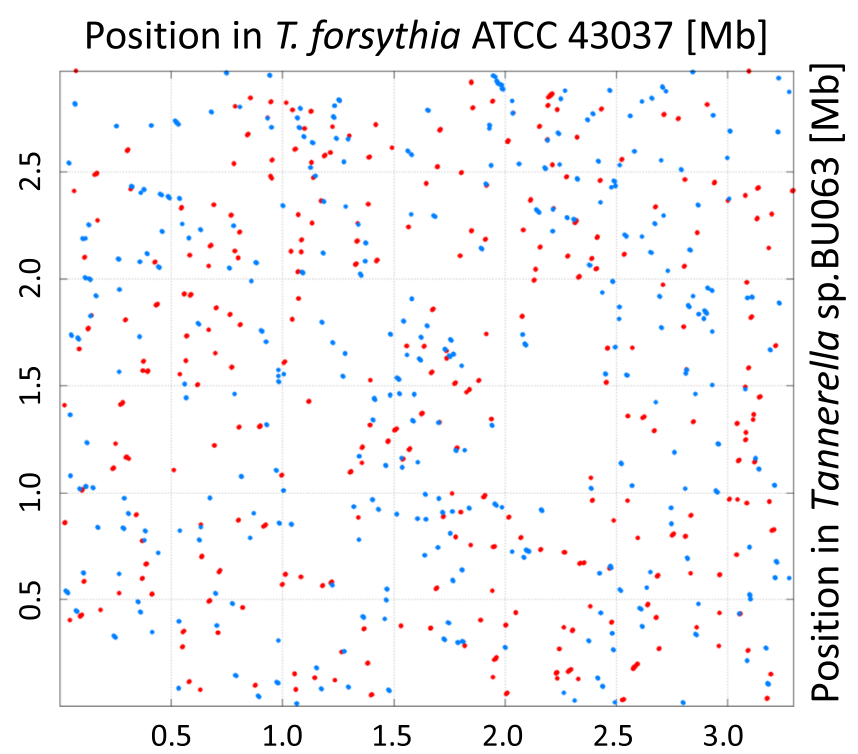

Fig. 4 Whole genome alignment between the six frame amino acid translations of both Tannerella sp. BU063 and the scaffolded and ordered ATCC 43037 assembly. Whereas the amino acid alignment reflects similarity with respect to gene content, the order of genes is not preserved

virulence and other phenotypical characteristics of strains 9610, UB20, 3313 may be required and that TF1589 may be of lower or no importance for the pathogenicity of $T$. forsythia.

Varying BSR values indicated sequence variation between different $T$. forsythia strains for the surface antigen BspA, one of the most comprehensively described virulence factors of $T$. forsythia and linked to pathogenesis by in vivo studies [2,3]. As noted previously [31], there were six putative BspA homologs predicted in the genome of strain $92 \mathrm{~A} 2$ besides the main BspA gene. For the gene most similar to the latter, termed BspA2, notable sequence variation was indicated as well. Both BspA and BspA2 showed BSR values close to zero (0.09 and 0.06, respectively) in Tannerella sp. BU063 indicating their absence. Variation was also found within the group of KLIKK proteases (i.e. miropsin-1, miropsin-2, mirolysin, mirolase, karilysin, forsilysin). In concordance with a previous study that described high-identity KLIKK protease homologs to be absent from Tannerella sp. BU063 but found a truncated mirolase-like open reading frame [18], we detected a homolog of mirolase with $46 \%$ sequence identity at $86 \%$ sequence coverage in the Tannerella sp. BU063 genome included here. As it was the best bidirectional hit, it is likely that it represents a true mirolase ortholog. The corresponding gene (NCBI protein database accession WP_069175679.1) is almost identical (97\% identity at $99 \%$ coverage) with the gene reported by Ksiazek et al. which was described to share a high degree of similarity in the catalytic domain with KLIKK proteases, but lacks a signal peptide, lacks an $\mathrm{N}$ terminal pro-fragment, and lacks the variable region that is characteristic of the C-terminal extension in KLIKK proteases.

In contrast to previous reports [28], we found a best bidirectional hit for karilysin in the Tannerella sp. BU063 assembly, in this case with $53 \%$ identity over the entire length of the gene. Within the work of Beall et al., only homology to the C-terminal part containing the secretion signal domain was reported. Interestingly, a large fraction of the dissimilarity between $T$. forsythia karilysin, as described by Ksiazek et al. [18], and the putative ortholog (NCBI protein database accession WP_083206853) identified in the gap-free Tannerella sp. BU063 genome assembly was found in regions other than the catalytic domain of the protein (Additional file 13: File S2).

Further work will be required to determine whether this gene is a bona fide functional karilysin ortholog. In any case, the previous conclusion that KLIKK proteases are completely absent from the Tannerella sp. BU063 genome has to be revised.

As potential targets for therapeutic strategies we would consider only those genes that are highly conserved in all $T$. forsythia strains, but absent or weakly conserved in the putative health-associated Tannerella sp. BU063 isolate, e.g. out of the 45 potential virulence factors the 20 genes showing a BSR of 0.9 or larger in $T$. forsythia strains and a BSR smaller than 0.6 in Tannerella sp. BU063 (Additional file 3: Table S3).

\section{Analysis of the T. forsythia pan-genome and comparison to Tannerella sp. BU063}

The comparison of gene repertoires encoded within different genomes of the same species has indicated 

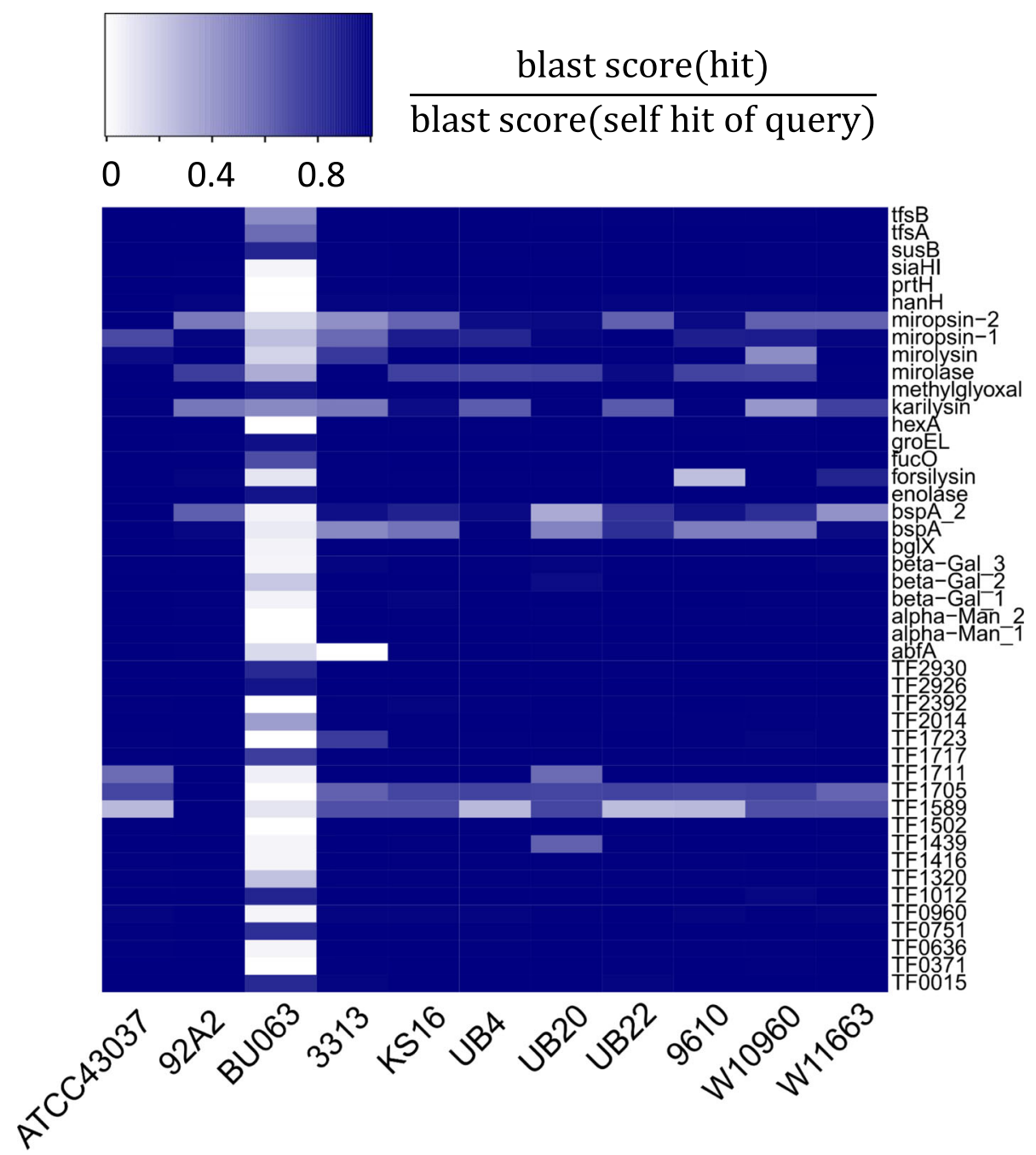

Fig. 5 Blast Score Ratio (BSR) values plotted as heatmap for 45 suggested virulence genes in ten T. forsythia strains and the genome of putative health-associated Tannerella sp. BU063. Gene sequences were blasted against the complete genomic sequences of each genome. Tannerella sp. BU063 achieved considerable BSR values for several genes that were actually suggested as virulence factors in pathogenic T. forsythia strains. On the other hand, some of the pathogenic strains show reduced similarity to some predicted virulence factors

remarkable flexibility $[35,36]$. For a particular species, a certain set of genes will be found in all of the studied genomes, while some genes will be restricted to just a subset thereof. The former genes will be referred to as the core genome, while the overall gene composition encompassing genes which may be present in just a single accession is called the pan-genome. Frequently, an extended version of the core genome is determined as well: As soon as genome comparisons take advantage of draft assemblies which may encompass gaps, the chance of finding additional core genes increases. Therefore, criteria are specified which demand core genes to be present in at least $80 \%$ or $90 \%$ of the studied genomes, respectively. Genes which meet such thresholds are assigned to a soft core genome. Based on the currently available annotated genomes of ten different strains of $T$. forsythia, i.e. ATCC 43037, 3313, KS16, UB4, UB20, UB22, 9610, WW11663, WW10960, and 92A2, we assessed a core genome of the species comprising 1864 genes, when requiring a core gene to be present in each strain without exception. Using less stringent criteria, further genes could be assigned as core genes. A soft core genome which required a gene to be present in $>=$ $90 \%$ of the strains contained 2043 genes; reducing the required threshold to $>=80 \%$, the number of genes increased to 2108. Analysis of the number of genes after iterative addition of the ten strains revealed saturation of the gene number in the core genome, whereas the pan 
genome of the species may still increase when analysing more strains (Fig. 6).

Of the genes found in the T. forsythia soft core genome (detection in $>=80 \%$ of strains), 894 were found to not possess an ortholog in the putative periodontal health-associated species Tannerella sp. BU063, hence, these genes will likely encompass genes that are associated with pathogenicity. Searching for Kyoto Encyclopedia of Genes and Genomes (KEGG) orthology terms overrepresented in this gene set turned out to be inconclusive, because such terms had been assigned to only about a quarter of the genes. We therefore adopted a different strategy to identify new candidate loci involved in the virulence of T. forsythia (see below).

Novel putative virulence factors and pathogenicity islands Genes involved in pathogenesis often co-localize in bacterial genomes within pathogenicity islands. Some strategies that aim at the identification of pathogenicity islands or other genomic islands make use of described structural elements that are common to such islands [37]. Here, we employed a comparative approach with respect to the Tannerella sp. BU063 genome to identify putative pathogenicity islands in T. forsythia strain 92A2. We defined a putative pathogenicity island as a genomic region in $T$. forsythia strain 92A2 that contained at least five consecutive genes, (i) which were part of the T. forsythia soft-core genome (detection in $>=80 \%$ of strains) and (ii) which could not be found in the Tannerella sp. BU063 genome.

In total, we detected 38 such regions, of which 18 encompassed at least seven consecutively ordered genes (Additional file 9: Table S9). Five of the island candidates contained more than ten such genes. Three of these regions are known from an initial genomic comparison between T. forsythia and Tannerella sp. BU063 [28], one further region corresponds to the sialic acid utilization locus [15]. Notably, nine of the islands encode SusD/TonB/TolClike components indicative of polysaccharide utilization loci (PULs). PULs are a unique feature of Bacteroidetes genomes encoding physically-linked carbohydrate-active enzymes next to an outer membrane transporter and a carbohydrate sensor/transcriptional activator and are important for colonization of nutritional niches [38, 39].

Three islands reported previously [28] were not detected by our approach. This discrepancy likely results from methodological differences: First, Beall et al. analysed a fragmented Tannerella sp. BU063 genome assembly derived from a single-cell genomics approach, whereas we took advantage of a gap-free genome assembly of Tannerella sp. BU063 generated after successful in vitro cultivation of this species. Second, in the previous reports all genes from T. forsythia strain 92A2 (misclassified as ATCC 43037) were considered, whereas we focused on genes of the T. forsythia soft core genome. Hence, the results by Beall et al. may include gene clusters derived from the 92A2 accessory genome (25\% of genes of a $T$. forsythia strain) and might not be representative for the species as a whole.

\section{Protein 0-glycosylation pathway genes}

The general protein $O$-glycosylation gene cluster of $T$. forsythia was described to contain a number of glycosyltransferases (Gtfs) and other enzymes involved in the biosynthesis of Tannerella's O-glycan structures [10]. However, the genes required for the initiation of the glycan synthesis have yet to be determined. Based on demonstrated analogies in the biosynthesis pathways of different bacterial glycoconjugates [40] it is conceivable that an initiating WbaP-like enzyme, like acting in the $\mathrm{O}$-antigen biosynthesis of Salmonella enterica [41], is also involved in O-glycan biosynthesis of T. forsythia. WbaP transfers galactose to an undecaprenyl-phosphate carrier residing within the inner membrane with the phosphate group facing the interior. Further sugar moieties are added to the nascent glycan structure from activated sugar precursors before it is flipped across the membrane by the flippase Wzx. Two such candidate genes were found in each T. forsythia strain included in this work. For the ATCC 43037 strain these were Tanf_04030 (WP_046824981.1), annotated
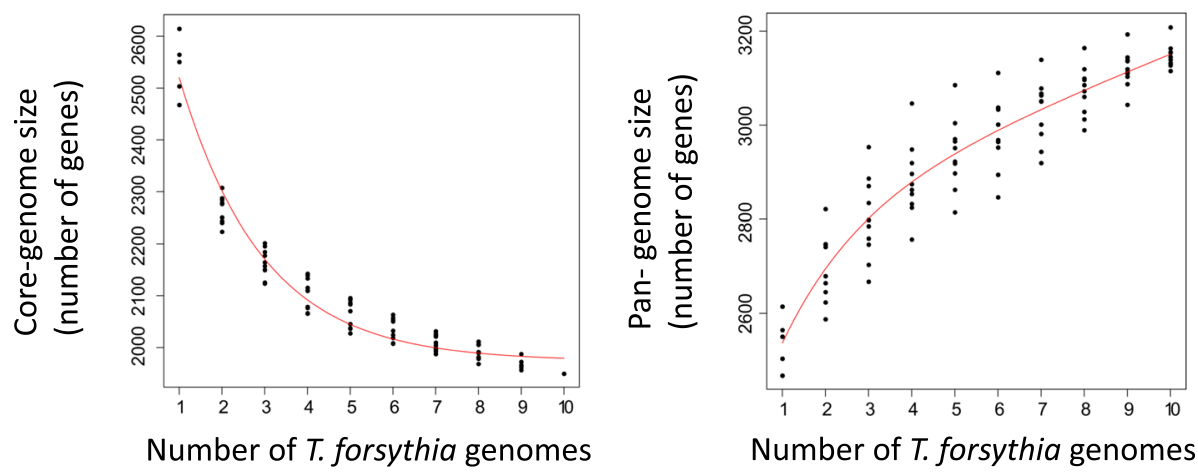

Fig. 6 Predicted core- and pan-genome sizes for T. forsythia based on ten genome assemblies using a sampling approach that iteratively adds genomes to the analysis. The species' core genome has a saturated size of 1900 genes, i.e. genes that are found to be conserved throughout the ten analysed strains are likely to be conserved throughout the whole species (left panel). In contrast, novel genes are expected to be found in newly sequenced $T$. forsythia genomes as indicated by the pan-genome curve that has not yet reached a saturation plateau (right panel) 
as a glycosyltransferase, and Tanf_09660 (WP_014226155.1), annotated as an undecaprenyl-phosphate glucose phosphotransferase. Both of these candidate genes had previously been knocked out individually, but no effect on $O$-glycan synthesis was observed (Gerald Posch, Bettina Janesch, and Christina Schäffer, unpublished data). There are numerous predicted Gtfs in the T. forsythia genome that are yet uncharacterized. Knock-out experiments for all of them would present a possible approach to further elucidate $O$ glycan biosynthesis, however, due to $T$. forsythia's slow growth and fastidious growth requirements, a rather tedious one. While it cannot be ruled out that the missing glycosylation pathway components are encoded by genes dispersed throughout the genome, it may be speculated that they are also co-located in a certain region. We searched for such putative glycosylation loci in the complete genome assembly of strain 92A2. In brief, a putative glycosylation locus was defined as an interval containing at least three predicted Gtfs or genes containing Gtf-associated domains within a stretch of 15 consecutive genes. Six such regions encompassing putative glycosylation loci could be discovered (Table 3), and one of them was found to be partially conserved in two different Parabacteroides genomes. Two regions, each, were found to be partially conserved in a single species, one in Bacteroides fragilis and the other one in Tannerella sp. BU063. These loci represent suitable starting points for further experimentation in order to confirm their role in glycosylation.

\section{Codon usage analysis}

The presence or absence of certain genes from the genomes of T. forsythia and Tannerella sp. BU063 may explain pathogenicity of the former and association with periodontal health of the latter. However, it is also possible that different expression levels of orthologous genes found in both genomes were responsible for the disease status. Further, genes that are highly expressed in T. forsythia may be assumed to be important for the species. For a number of microorganisms it has been shown that expression levels of individual genes can be predicted based on the differential usage of synonymous codons within the genes [42, 43]. Whereas compositional constraints such as GC content are believed to be responsible for shaping codon usage in many genomes throughout different domains of life, analysis of codon usage is especially interesting in prokaryotes, where the differential usage of synonymous codons of some genomes has been shown to correlate with the availability of the corresponding tRNAs in the cell. The frequent codon/ tRNA pairs are thought to enable fast translation of these regions, whereas rare codon/tRNA pairs can slow down translation and improve accuracy. Together, this is often referred to as "translational optimization". Based on these findings, different approaches have been developed that try to predict expressivity of a gene, based on the codon usage bias found in its sequence.

We analysed the effective number of codons $(\mathrm{Nc})$ values for the genomes of T. forsythia ATCC 43037 and Tannerella sp. BU063 and their relationship to the frequency of $\mathrm{G}$ and $\mathrm{C}$ at synonymous 3rd codon positions (GC3s-content) (Fig. 7). The maximum possible Nc value is 61 , as it represents a case whereby all 61 (non-stop) codons are used equally. This is only possible at balanced GC3s content; deviations from that balance result in lower maximum possible Nc values. Our analysis indicated a bias in codon usage for both T. forsythia ATCC 43037 and Tannerella sp. BU063 (Additional file 5: Tables S5, Additional file 6: Table S6, Additional file 7: Table S7 and Additional file 8: Table S8). While for many genes the Nc value is roughly in the predicted range, numerous genes display a codon usage bias that cannot be explained by compositional constraints alone and, hence, may be explained by translational optimization. In the absence of knowledge on gene expression levels in T. forsythia, we used two self-consistent indices, self-consistent Codon Adapation Index (scCAI) [44] and self-consistent normalized Relative Codon Adaption (scnRCA) [45], in combination with criteria that can be applied to the results of both approaches to predict the nature of this bias [46]. scCAI detected the most prominent codon usage bias, predicted to be shaped by GC3s content, in both T. forsythia and Tannerella sp. BU063 with content criteria values of 0.85 and 0.89 , respectively, both above the proposed threshold of 0.7 (Additional file 5: Table S5, Additional file 7: Table S7). The similar scnRCA index aims at predicting a potential translational bias. In case of $T$. forsythia, the scnRCA method was able to remove the influence of the GC3s bias on the analysis as indicated by a content criterion value of 0.56 (Additional file 6: Table S6, Additional file 8: Table S8). However, a content criterion value $>0.5$ is suggested to indicate a bias shaped by a GC skew. Two criteria that would indicate a translational bias, the ribosomal criterion and the strength criterion, were both negative. For Tannerella sp. BU063, scnRCA was not able to remove the GC3s bias sufficiently, as the remaining bias is still predicted to be shaped by GC3s content (content criterion >0.7) This matches the observation of a higher GC3s content in Tannerella sp. BU063 when compared to T. forsythia (Fig. 7). We conclude that compositional constraints are the main factors shaping the codon usage bias in both T. forsythia and Tannerella sp. BU063. Whether translational optimization is also a factor shaping the biases in one or both of the genomes remains to be elucidated.

\section{Discussion}

We were able to assemble $99 \%$ of the T. forsythia ATCC 43037 genome into only three fragments by using the existing contigs generated by Friedrich et al. [20] and new mate- 
Table 3 Positions of putative glycosylation (PGL) loci in T. forsythia strain FDC 92A2

\begin{tabular}{|c|c|c|c|c|c|}
\hline Locus tag (RefSeq, GenBank) & Position & Strand & Protein ID, Description & Conserved Domains & $\mathrm{dbCAN}$ \\
\hline \multicolumn{6}{|l|}{ PGL_1 } \\
\hline BFO_RS00485, BFO_0104 & $119,936-121,180$ & + & $\begin{array}{l}\text { WP_014223582.1, glycosyltransferase } \\
\text { group } 1 \text { family protein }\end{array}$ & $\begin{array}{l}\text { cl28208 } \\
\text { RfaB superfamily }\end{array}$ & GT4 \\
\hline BFO_RS00535, BFO_0114 & $132,395-133,165$ & + & WP_014223590.1, hypothetical protein & $\begin{array}{l}\text { cl11394 } \\
\text { Glyco_tranf_GTA_type superfamily }\end{array}$ & - \\
\hline BFO_RS00545, BFO_0116 & $133,715-134,380$ & + & WP_041590509.1, hypothetical protein & $\begin{array}{l}\text { cl11394 } \\
\text { Glyco_tranf_GTA_type superfamily }\end{array}$ & - \\
\hline BFO_RS00550, BFO_0117 & $134,417-135,097$ & + & WP_014223593.1, glycosyl transferase & $\begin{array}{l}\text { cl01298 } \\
\text { Glyco_transf_25 superfamily }\end{array}$ & GT25 \\
\hline \multicolumn{6}{|l|}{ PGL_2 } \\
\hline BFO_RS02100, BFO_0467 & $500,734-501,384$ & - & WP_052299218.1, hypothetical protein & $\begin{array}{l}\text { cl02988 } \\
\text { Glyco_transf_10 superfamily }\end{array}$ & GT10 \\
\hline BFO_RS02105, BFO_0468 & $502,333-504,648$ & - & $\begin{array}{l}\text { WP_014223924.1 } \\
\text { penicillin-binding protein 1C }\end{array}$ & $\begin{array}{l}\text { TIGR02073 } \\
\text { PBP_1C }\end{array}$ & GT51 \\
\hline BFO_RS02135, BFO_0475 & $513,630-514,787$ & + & $\begin{array}{l}\text { WP_014223931.1 } \\
\text { mannosyltransferase }\end{array}$ & $\begin{array}{l}\text { cd03809 } \\
\text { GT1_mtfB_like }\end{array}$ & GT4 \\
\hline \multicolumn{6}{|l|}{ PGL_3 } \\
\hline BFO_RS02420, BFO_0544 & $586,421-587,608$ & + & $\begin{array}{l}\text { WP_014223997.1, glycosyl transferase } \\
\text { family } 1\end{array}$ & $\begin{array}{l}\text { cl10013 } \\
\text { Glycosyltransferase_GTB_type }\end{array}$ & GT4 \\
\hline BFO_RS02430, BFO_0547 & $588,656-589,774$ & + & $\begin{array}{l}\text { WP_014223999.1, glycosyl transferase } \\
\text { family } 1\end{array}$ & $\begin{array}{l}\text { cl10013 } \\
\text { Glycosyltransferase_GTB_type }\end{array}$ & GT4 \\
\hline BFO_RS02435, BFO_0564 & $589,763-590,959$ & - & WP_014224000.1, hypothetical protein & $\begin{array}{l}\text { cl10013 } \\
\text { Glycosyltransferase_GTB_type }\end{array}$ & - \\
\hline \multicolumn{6}{|l|}{ PGL_4 } \\
\hline BFO_RS07405, BFO_1699 & $1,808,692-1,809,366$ & - & WP_014225043.1, glycosyl transferase & $\begin{array}{l}\text { cl01298 } \\
\text { Glyco_transf_25 superfamily }\end{array}$ & - \\
\hline BFO_RS07410, BFO_1700 & $1,809,356-1,810,438$ & - & WP_014225044.1, glycosyl transferase & $\begin{array}{l}\text { cl10013 } \\
\text { Glycosyltransferase_GTB_type }\end{array}$ & GT4 \\
\hline BFO_RS14425, BFO_1705 & $1,812,769-1,814,883$ & - & WP_052299248.1, hypothetical protein & $\begin{array}{l}\text { cd03801 } \\
\text { GT1_YqgM_like }\end{array}$ & GT4 \\
\hline \multicolumn{6}{|l|}{ PGL_5 } \\
\hline BFO_RS08625, BFO_1977 & $2,106,020-2,107,996$ & - & WP_014225302.1, hypothetical protein & $\begin{array}{l}\text { cl28208 } \\
\text { RfaB superfamily }\end{array}$ & - \\
\hline BFO_RS08630, BFO_1978 & $2,108,002-2,108,745$ & - & $\begin{array}{l}\text { WP_014225303.1, glycosyl transferase } \\
\text { family } 2\end{array}$ & $\begin{array}{l}\text { cd04179 } \\
\text { DPM_DPG-synthase_like }\end{array}$ & GT2 \\
\hline BFO_RS14090, BFO_1987 & $2,122,302-2,123,087$ & + & WP_052299260.1, hypothetical protein & $\begin{array}{l}\text { cd04186 } \\
\text { GT_2_like_c }\end{array}$ & GT2 \\
\hline BFO_RS08670, BFO_ & $2,123,084-2,124,346$ & + & WP_041590821.1, hypothetical protein & $\begin{array}{l}\text { cl10013 } \\
\text { Glycosyltransferase_GTB_type }\end{array}$ & GT4 \\
\hline BFO_RS08675, BFO_1990 & $2,124,694-2,126,031$ & + & $\begin{array}{l}\text { WP_014225312.1, glycosyltransferase } \\
\text { group } 1 \text { family protein }\end{array}$ & $\begin{array}{l}\text { cl10013 } \\
\text { Glycosyltransferase_GTB_type }\end{array}$ & GT4 \\
\hline BFO_RS08680, BFO_1989 & $2,126,026-2,127,159$ & - & WP_014225313.1, glycosyl transferase & $\begin{array}{l}\text { cl10013 } \\
\text { Glycosyltransferase_GTB_type }\end{array}$ & GT4 \\
\hline \multicolumn{6}{|l|}{ PGL_6 } \\
\hline BFO_RS10550, BFO_2565 & $2,598,381-2,599,619$ & - & WP_014225708.1, hypothetical protein & $\begin{array}{l}\text { cl10013 } \\
\text { Glycosyltransferase_GTB_type }\end{array}$ & GT4 \\
\hline BFO_RS10555, BFO_2566 & $2,599,616-2,600,713$ & - & $\begin{array}{l}\text { WP_014225709.1, UDP-N-acetylglucosamine } \\
\text { 2-epimerase (non-hydrolyzing) }\end{array}$ & $\begin{array}{l}\text { cd03786 } \\
\text { GT1_UDP-GIcNAc_2-Epimerase }\end{array}$ & - \\
\hline BFO_RS10600, BFO_2575 & $2,607,474-2,608,256$ & + & $\begin{array}{l}\text { WP_014225718.1 } \\
\text { glycosyl transferase }\end{array}$ & $\begin{array}{l}\text { cd04179 } \\
\text { DPM_DPG-synthase_like }\end{array}$ & GT2 \\
\hline
\end{tabular}




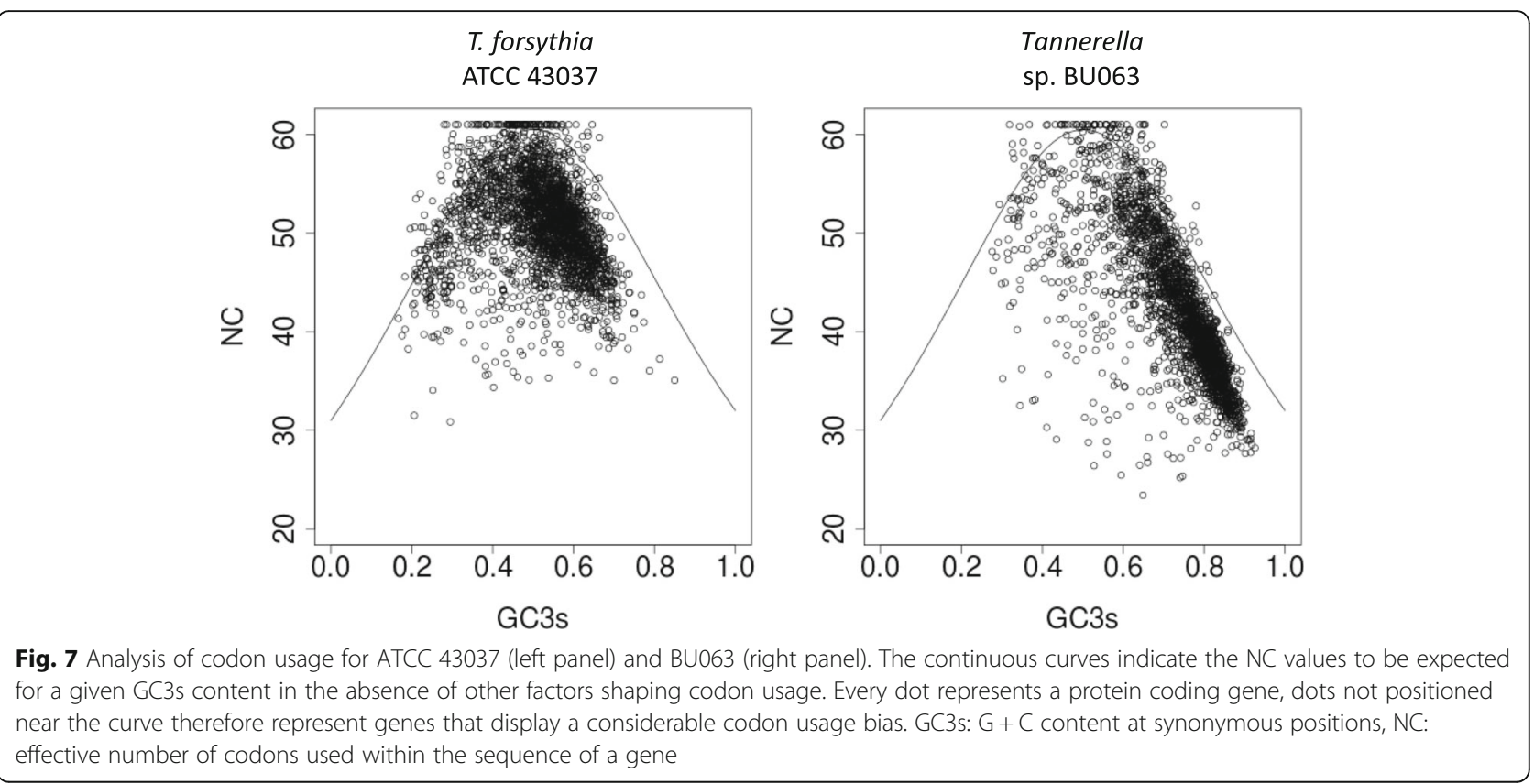

pair data of about 800-fold genome coverage. For obtaining an assembly in one uninterrupted sequence for the T. forsythia reference strain the use of sequencing technologies that provide medium-sized or long reads will be required since short-reads, even at very high coverage, were not sufficient to close all gaps. This finding is consistent with the fact that the two completely contiguous $T$. forsythia assemblies for strains KS16 and 3313 had been generated employing Sanger sequencing data in combination with short-read data. However, our current assembly result for ATCC 43037 represents an almost complete genome sequence as a valuable resource for $T$. forsythia studies.

In comparative analyses we provided an assessment of the presence or absence of currently known as well as suggested virulence factors in all presently available $T$. forsythia genome assemblies. We may have missed orthologs in a given strain if genes were located in a region of the genome that was not covered by its assembly. However, our results largely extend previous findings by Endo et al. [22], that showed a small subset of the genes included in our analysis to be conserved in strains $92 \mathrm{~A} 2$ (mistakenly referred to as ATCC 43037 by the authors), KS16, and 3313, as well as in 16 other T. forsythia strains. Similarly, our gene numbers for the core genome were slightly higher than previously estimated by Endo et al., who reported a core genome size of 1733 genes. The deviation, however, is to be expected as only three of the $19 \mathrm{~T}$. forsythia strains included in their work had complete genome assemblies whereas 16 were incompletely assembled. Hence, a bona fide core gene that was located in a region uncovered in one or more of these incomplete assemblies, would not be included; an effect that can be assumed to be stronger, the higher the number of included incomplete genomes is.

We suggested a number of regions that may be considered as pathogenicity islands. It should be noted that the term "pathogenicity island" usually refers to a genomic region containing genes that were introduced by horizontal gene transfer (HGT) [47]. Our approach does not consider the latter requirement, i.e. we did not assess whether the detected regions show traces of HGT. Further, the genes contained within putative pathogenicity islands presented in our work were inferred to be possibly linked to pathogenicity based on their presence in the $T$. forsythia core genome and their absence in Tannerella sp. BU063. How many and which of the reported candidate regions represent true pathogenicity islands, in the sense of the common definition, has yet to be discovered and will require experimental verification. In the context of periodontitis research, however, the finding that such a region is involved in pathogenesis is relevant, regardless of whether or not HGT took place.

In strain 92A2 we detected the already known glycosylation locus and confirmed that parts of it were shared throughout numerous Bacteroidales species. We speculate that the glycosylation pathway genes that synthesize the core of the glycan are organized in a different and less conserved way than the already described parts that assemble the outer part of the glycan. It should be noted that our approach relied on the current annotation of $T$. forsythia genes, their predicted functions, and their conserved domains. It is therefore possible that some carbohydrateactive genes were not included in the analysis simply because this functionality has not yet been predicted. 
Especially as research on prokaryotic glycosylation pathways is still vastly expanding, it will be interesting to see if more conclusive results will be obtainable in the future, as the knowledge on protein architecture and the conserved domain functions stored therein increases.

\section{Conclusion}

The involvement of Tannerella forsythia in periodontal disease manifests a major challenge to national health systems. In this work, we provide molecular resources which will facilitate future work on T. forsythia. We provide an improved genome assembly of the reference type strain $T$. forsythia ATCC 43037, and we define a soft-core genome and an accessory genome of the species. Comprehensive characterization of the $T$. forsythia genome relative to the non-pathogenic isolate Tannerella sp. BU063 allowed us to confirm known virulence factors or suggest their reevaluation, respectively. Importantly, we highlight genes which so far have not been implicated in the pathogenesis of $T$. forsythia. In summary, our work provides new perspectives for work on Tannerella biology, for both basic as well as applied research.

\section{Methods}

\section{DNA source, extraction and quality control}

The type strain of T. forsythia (ATCC 43037 = FDC 338) was obtained from ATCC (Manassas, VA, USA) and grown under anaerobic conditions in brain-heart infusion broth with supplements as described previously [12]. Bacterial DNA was extracted using the GeneElute Bacterial Genomic DNA Kit (Sigma-Aldrich, Vienna, Austria) following the manufacturer's protocol. The quality of the genomic DNA was checked on a $0.6 \%$ standard agarose gel stained with ethidium bromide, and using a NanoDrop ND-1000 spectrophotometer (ThermoFisher, Waltham, MA, USA). Quantification was performed using a Qubit 3.0. fluorometer together with a dsDNA BR assay kit (ThermoFisher, Waltham, MA, USA).

\section{Mate-pair library preparation and sequencing}

Starting from $1 \mu \mathrm{g}$ of genomic DNA, a mate-pair library was prepared using a Nextera mate-pair library preparation kit (FC-132-1001, Illumina, San Diego, CA, USA) applying the gel-free version of the library preparation protocol, according to the supplier's instructions. Briefly, the protocol consists of tagmentation, strand displacement, AMPure purification of the strand displacement reaction, and circularization. After linear DNA digestion, circularized DNA was sheared to a size of 300-1000 bp with a Covaris S220 instrument (Covaris, Woburn, MA, USA) and the following settings: $40 \mathrm{~s}$ at $20 \%$ duty cycle, intensity 50 , temperature $6^{\circ} \mathrm{C}$ and 200 cycles per burst. Next, sheared DNA fragments containing the biotinylated junction adapter were purified using streptavidin magnetic beads followed by end-repair, A-tailing, and ligation of Illumina adapters to the ends of the DNA fragments. The library was amplified by polymerase chain reaction (PCR) applying the following cycling conditions: initial denaturation at $98^{\circ} \mathrm{C} / 30 \mathrm{~s}$, followed by 10 cycles at $98^{\circ} \mathrm{C} / 10$ s, $60^{\circ} \mathrm{C} / 30 \mathrm{~s}, 72^{\circ} \mathrm{C} / 30 \mathrm{~s}$, and a final extension at $72{ }^{\circ} \mathrm{C} / 5 \mathrm{~min}$. After PCR clean-up, $1 \mu \mathrm{l}$ of the library was taken for validation using a 2100 Bioanalyzer (Agilent, Santa Clara, CA, USA). Library quantification was accomplished on a Qubit 3.0 fluorometer using a dsDNA BR assay kit, thereafter, the library was sequenced at the VBCF Next Generarion Sequencing core facility (Vienna, Austria) on an Illumina HiSeq 2500 sequencing instrument using v4 sequencing chemistry and a $2 \times 125 \mathrm{nt}$ paired-end sequencing protocol.

\section{Quality control and filtering of Illumina sequencing data}

FastQC v0.11.4 (http://www.bioinformatics.babraham.ac. $\mathrm{uk} /$ projects/fastqc/) was used for initial quality checking of raw sequencing reads and to assess the outcome of read filtering procedures. Raw reads from the paired-end library were de-duplicated, considering two read pairs as duplicates if bases 15 to 50 of both the forward and of the reverse reads were identical. The non-redundant reads were then trimmed and filtered with Trimmomatic 0.35 [48], applying the following parameters: LEADING: 3 TRAILING:3 SLIDINGWINDOW:4:15 MINLEN:36. Due to remaining potential quality issues the reads were additionally cropped on both ends (15 bases at the head, eight bases at the tail), using the fastx toolkit (http://hannonlab.cshl.edu/fastx_toolkit/). Raw reads from the matepair library were cropped to various lengths for scaffolding test runs (only using nucleotides $1-50,1-80$ or $8-106$, of each read, respectively); for the final scaffolding procedure the $50 \mathrm{nt}$ long cropped reads were used.

\section{Assembly scaffolding}

SOAPdenovo 2.04 [49] was used for scaffolding. The helper program finalFusion, also maintained by SOAPdenovo developers, was used to prepare the input contigs for applying the scaffolding steps (map-scaff) of the main program. The $\mathrm{k}$-mer size parameter was varied in repetitions of this procedure $(K=33,43,45,47,49)$, where $K=47$ resulted in an assembly with the highest values for N50 scaffold length, and for the size of the largest scaffold, respectively. Gap-filling, as carried out by SOAPdenovo, was enabled by using the $-\mathrm{F}$ parameter. Results were assessed using QUAST v3.2 [50], additionally, critical links were validated by manual inspection of the mate-pairs supporting these links in IGV $2.3 .68[51,52]$ and by analysing the amount and mapping positions of mate-pairs supporting these links.

\section{Phylogenetic analysis}

Mash v2.0 [30] was used for distance calculation (programm call "mash dist" with default parameters). Trees 
were calculated using the Fitch-Margoliash algorithm as implented in PHYLIP v3.6 [53], with global rearrangement and randomizing the input order (10x jumbling). Trees were displayed using Newick utilities [54].

\section{Whole-genome alignments}

Whole-genome alignments of more than two genomes were generated and visualized with Mauve (version snapshot 2015-02-13 build 0) [55], using the progressiveMauve algorithm with default parameters --seed-weight $=15$--gapopen $=400 \quad$-gap-extend $=30 \quad-$-scoring-scheme $=$ sp. The contigs of fragmented assemblies were ordered and oriented using the contiguous genome assembly of $T$. forsythia 92A2 as reference with Mauve's "reorder contigs" module prior to alignment. Additional alignments employing blastn $[56,57]$ were used for calculating the fraction of alignable regions to strain $92 \mathrm{~A} 2$ per genome.

Whole-genome alignments between ATCC 43037 and BU063 were carried out using the MUMmer 3.23 software package [58], employing nucmer with default parameters --mumreference -b 200 --nobanded -c 65 --delta -D 5 -d 0.12 --extend -g 90 -1 20 --optimize --simplify for aligning and mummerplot with the option --filter for creating Gnuplot scripts. Six-frame amino acid translation alignments between ATCC 43037 and BU063 were generated with promer with the parameters -mumreference -b 60 -c 20 -g 30 -1 6 -m 8 - $\times 2$. Gunplot scripts were adapted manually with respect to aesthetics and readability and plotted with Gnuplot 4.4 (http://www.gnuplot.info/).

\section{Analysis of core- and accessory genomes}

The T. forsythia core and accessory genomes were compiled using components of the GET_HOMOLOGUES pipeline [59]. All genome assemblies included in the analysis were downloaded as RefSeq gbff files from the NCBI $\mathrm{ftp}$ server (ftp://ftp.ncbi.nlm.nih.gov/genomes/) as input for get_homologues.pl. As starting point for further analysis, an all-vs-all blastp [56, 57] of all coding sequences (CDS) contained in the input files was performed in a batchwise manner, using the parameters -dbsize 100,000, 000 -seg yes -soft_masking true -evalue 0.01 -outfmt 6 qseqid sseqid pident length qlen slen qstart qend sstart send evalue bitscore -max_target_seqs $\mathrm{N}$ (where $\mathrm{N}$ is the total number of sequences in the database used in that run). Based on the blastp results, clusters of putative orthologs were clustered with get_homologues.pl in two parallel runs, one employing the OrthoMCL algorithm [60], the other employing the cluster of orthologous groups of proteins (COG) triangles algorithm [61]. Both algorithms infer orthology based on bidirectional best hits (sometimes also referred to as symmetrical best hits or reciprocal best hits). Additional thresholds for two genes to be allowed to group in the same cluster were: sequence identity of at least $30 \%(-S 30)$, sequence coverage of the alignment of at least 75\% (-C 75), and an Expect (E) value of $<10^{-5}$ (-E 1e-05). Clusters were allowed to contain genes from any number of the included genomes $(-t)$ ). For further parameters the default values $-\mathrm{c} 0-\mathrm{z} 0-\mathrm{I} 0-\mathrm{m}$ local -n 2 -M 0 -G 1 -P 0 -F 1.5 -N 0 -B 50 -b 0 -s 0 -D 0 -g 0 -a ' 0 ' $-\times 0$ - R 0 -A 0 were used. The genome of strain 92A2 was used as reference $(-r)$, which, however, only has an influence on the names given to the resulting clusters when using OrthoMCL or COG triangles as clustering method. The intersection of the cluster sets generated by the two different algorithms was extracted using compare_clusters.pl and used for further analysis. Orthology clusters containing genes from all $T$. forsythia genomes were extracted using the script parse_pangenome matrix.pl; the genes contained in these clusters constitute the core genome of $T$. forsythia. In an additional run, orthologs were required to be present in at least $80 \%$ of the $T$. forsythia genomes to become part of a relaxed form of the core genome, sometimes referred to as "soft core genome". Allowing an ortholog to be absent in one of the assemblies reduces the risk of incorrectly excluding bona fide core genes from the core genome due to annotation, assembly or sequencing errors, or the incomplete nature of some of the included assemblies. Tannerella forsythia core and pan genome sizes were estimated based on random sampling by using only the $T$. forsythia genomes as input for get_homologues.pl with the additional parameter -c. Plots illustrating these estimations were generated with plot_pancore_matrix.pl, using the parameter -f core_Tettelin for the core genome plot and -f pan for the pangenome plot. Comparisons on the presence or absence of single genes were carried out using the script check BDBHs.pl. All these Perl scripts are part of the GET_HOMOLOGUES pipeline.

\section{Detection of putative pathogenicity islands}

Based on the results of the pan-genome analysis, putative pathogenicity islands were detected as follows: Genes that were found to be present in at least eight of the ten T. forsythia strains but absent from Tannerella sp. BU063 were considered to be T. forsythia-specific and assessed for their co-localization within the genome of strain 92A2 using the Perl script GeneClusterFinder.pl developed in this work. The script takes a file containing all annotated genes of the genome in tabular form (as can be downloaded from https://www.ncbi.nlm.nih.gov/ genome/proteins/11045?genome_assembly_id=231734)

as reference input file, assigning sequential numbers to the genes sorted by position. This step is necessary, as the locus tags used by NCBI are not necessarily sequential. A file containing the locus tags of all $T$. forsythiaspecific genes is then loaded as second input and the corresponding sequential numbers are extracted. Finally, 
stretches of consecutive numbers are searched in the resulting set of numbers.

\section{Searching for glycosylation loci}

Putative glycosylation loci were defined as genomic regions where at least three predicted Gtfs or other putative glycosylation-related genes occur within a stretch of 15 consecutive genes. The current Reference Sequence (RefSeq) gene set for strain 92A2 was used as reference. The complete gene set was annotated using the carbohydrate-active enzyme specific annotation web server dbCAN [62]. Additionally, conserved domains were annotated for all genes, using NCBI's CD-Search [63-66] (parameters: database: CDD 53,069 PSSMs, expect value threshold 0.01, compositioncorrected scoring on, low-complexity filter off, maximum number of hits 500, include retired sequences on). All genes that either were predicted as Gtfs by dbCAN or predicted to contain a Gtf-associated conserved domain by CD-Search were combined and used for the subsequent steps. Analysing the co-localization of these genes employed a custom Perl script implementing a sliding-window approach to find stretches of 15 consecutive genes encompassing at least three putative Gtfs. Redundant windows were removed and overlapping ones combined; the split region of the circular genome in the assembly was checked manually. Additional analysis on the presence or absence of the detected putative glycosylation loci in other members of the Bacteroidales order was performed using Gecko 3.1 [67]. Organisms included in this analysis were chosen based on previous work [11], the RefSeq assembly versions of the corresponding genomes were downloaded from the NCBI ftp server as GenBank flat files (Additional file 4: Table S4) and used as input for the script gecko3_gb_ to_transclust_to_cog.py from the Gecko suite. First, this script was used to prepare a Blastp search (version 2.2.30+) by using the parameter -prepareSingleBlast. This sets up a database and a query FASTA file containing all CDS from all included genomes and executes the corresponding Blastp command with an E-value cut-off of 0.01 . The file containing the Blastp results as well as the query file described above were loaded into Transclust 1.0 [68]. Clustering was performed using Best $\mathrm{Hit}(\mathrm{BeH})$ as cost model and a density parameter of 40 . The results produced by Transclust were converted into the format required as input for Gecko, using the script gecko3_gb_ to_transclust_to_cog.py with the parameter -transclustTo$\mathrm{Cog}$. Whether a putative glycosylation locus or parts of it can be found in one of the included genomes apart from T. forsythia was checked as follows: The homology IDs assigned to the genes constituting such a locus during data preparation were extracted and used as query cluster in a "manual cluster" search in Gecko 3.1. The number of genes required in a cluster to be detected was initially set to the number of genes in the query minus one and the maximum distance between genes was set to 15 . If this did not detect the cluster or parts of it in non- $T$. forsythia genomes, additional searches were carried out with more relaxed parameters, i.e. setting the maximum distance between genes to 30 and reducing the minimum number of genes required in a cluster to be detected. The value for the latter parameter was decreased by one in each subsequent run either until parts of the cluster were found in a non-T. forsythia strain or until the value was two.

\section{Codon usage analysis}

Nc values [69] and GC3s values were calculated with CodonW using the parameters -all_indices -nomenu (http:// codonw.sourceforge.net//culong.html). Fasta files containing the nucleotide sequences of all CDS of the respective genome were downloaded from NCBI's ftp server and all CDS for which the "pseudo" qualifier was set to "true" were eliminated prior to the analysis. scCAI values and scnRCA values were calculated using the program scnRCA with the parameters -g true $-\mathrm{d} 2.0$-p $1.0-\mathrm{m}-1$ and GenBank flat files as input files $[44,45]$.

\section{Supplementary information}

Supplementary information accompanies this paper at https://doi.org/10. 1186/s12864-020-6535-y.

Additional file 1: Table S1. $\times$ Blast Score Ratio values of 45 suggested virulence genes blasted against entire Tannerella genomes (heatmap plot in Fig. 4).

Additional file 2: Table S2. Blast Score Ratio values of 45 suggested virulence genes blasted against annotated Tannerella CDS (heatmap plot in Additional file 11: Figure S2).

Additional file 3: Table S3. List of promising therapeutic targets based on presence-absence analyses.

Additional file 4: Table S4. Bacteroidales genome assemblies used for the identification of putative glycosylation loci.

Additional file 5: Table S5. Codon usage bias (scCAl). Top 20 genes of ATCC 43037 (a) and Tannerella sp. BU063 (b) showing the highest scCAl values. Only functionally annotated proteins were selected.

Additional file 6: Table S6. Codon usage bias (scnRCA). Top 20 genes of ATCC 43037 (a) and Tannerella sp. BU063 (b) showing the highest scnRCA values. Only functionally annotated proteins were selected.

Additional file 7: Table S7. Codon usage bias (scCAl) including "hypothetical proteins". Top 20 genes of ATCC 43037 (a) and Tannerella sp. BU063 (b) showing the highest scCAl values.

Additional file 8: Table S8. Codon usage bias ( $\operatorname{scnRCA}$ ) including "hypothetical proteins". Top 20 genes of ATCC 43037 (a) and Tannerella sp. BU063 (b) showing the highest scnRCA values.

Additional file 9: Table S9. Pathogenicity islands in T. forsythia strain 92A2 as inferred from comparisons to Tannerella sp. BU063 and positions of genes encoded therein including their functional annotation.

Additional file 10: Figure S1. Span size distribution of the mate-pair library prepared from DNA of T. forsythia strain ATCC 43037. The peak of the distribution is at $1759 \mathrm{bp}$, indicated by the red line.

Additional file 11: Figure S2. Blast Score Ratio values plotted as heatmap for 45 suggested virulence genes in ten T. forsythia strains and the genome of a putative health-associated Tannerella sp. BU063. In contrast to Fig. 4, the 45 gene sequences were blasted against sequences of annotated CDS in each genome. 
Additional file 12: File S1. Alignments of the sequence KP715369 to T. forsythia genomes.

Additional file 13: File S2. Detailed view on the alignment of $T$. forsythia karilysin to a putative orthologue in Tannerella sp. BU063.

\section{Abbreviations}

ATCC: American Type Culture Collection; BeH: Best hit; BSR: BLAST score ratio; CDS: Coding sequence; COG: Cluster of orthologous groups of proteins: CTD: C-terminal domain; E-value: Expect value; GC3s: $\mathrm{G}$ and $\mathrm{C}$ at synonymous 3rd codon positions; Gtf: Glycosyltransferase; HGT: Horizontal gene transfer; IgSF: Immunoglobulin-superfamily; kbp: Kilobasepair; KEGG: Kyoto encyclopedia of genes and genomes; Mbp: Megabasepair; Nc: Effective number of codons; NCBI: National Center for Biotechnology Information; nt: Nucleotides; PCR: Polymerase chain reaction; PUS: Polysaccharide utilization locus; RefSeq: Reference Sequence; scCAl: Self-consistent Codon Adapation Index; scnRCA: Self-consistent normalized Relative Codon Adaption; S-layer: Surface layer; T9SS: Type IX secretion system

\section{Acknowledgements}

We are grateful to Valentin Friedrich for providing a DNA sample from the ATCC 43037 type strain. Part of the Illumina sequencing data used in this study were generated at the VBCF NGS Unit (www.viennabiocenter.org/ facilities). The computational results presented have been achieved in part using the Vienna Scientific Cluster (VSC).

\section{Authors' contributions}

NFZ analysed the data and drafted the manuscript. NSP performed wet-lab experiments. JCD and $\mathrm{HH}$ supervised the study and revised the manuscript with contributions from CS. All authors read and approved the final manuscript.

\section{Funding}

This work was supported by the Austrian Science Fund (FWF) project P26836-B22 (to CS) and the Universität für Bodenkultur, Wien. The funding bodies played no role in the design of the study and collection, analysis, and interpretation of data and in writing the manuscript.

\section{Availability of data and materials}

The improved genome assembly for T. forsythia ATCC 43037 has been deposited at DDBJ/ENA/GenBank under the accession VFJI00000000. The version described in this paper is version VFJI01000000. Mate-pairs of T. forsythia ATCC 43037 were deposited in the Sequence Read Archive under accession SRR9302598 (BioProject PRJNA548889, BioSample SAMN12058270).

\section{Ethics approval and consent to participate}

Not applicable.

\section{Consent for publication}

Not applicable.

\section{Competing interests}

$\mathrm{HH}$ is a member of the editorial board of BMC Genomics.

\section{Author details}

${ }^{1}$ Department of Biotechnology, Institute of Computational Biology, University of Natural Resources and Life Sciences (BOKU), Vienna, Austria. ${ }^{2}$ Department of NanoBiotechnology, NanoGlycobiology unit, University of Natural Resources and Life Sciences (BOKU), Vienna, Austria.

Received: 18 June 2019 Accepted: 23 January 2020

\section{Published online: 11 February 2020}

\section{References}

1. Hajishengallis G, Lamont RJ. Beyond the red complex and into more complexity: the polymicrobial synergy and dysbiosis (PSD) model of periodontal disease etiology. Mol Oral Microbiol. 2012;27:409-19.

2. Sharma A, Sojar HT, Glurich I, Honma K, Kuramitsu HK, Genco RJ. Cloning, expression, and sequencing of a cell surface antigen containing a leucinerich repeat motif from Bacteroides forsythus ATCC 43037. Infect Immun. 1998;66:5703-10.
3. Sharma A, Inagaki S, Honma K, Sfintescu C, Baker PJ, Evans RT. Tannerella forsythia-induced alveolar bone loss in mice involves leucine-rich-repeat BspA protein. J Dent Res. 2005;84:462-7.

4. Saito T, Ishihara K, Kato T, Okuda K. Cloning, expression, and sequencing of a protease gene from Bacteroides forsythus ATCC 43037 in Escherichia coli. Infect Immun. 1997:65:4888-91.

5. Lee S-W, Sabet M, Um H-S, Yang J, Kim HC, Zhu W. Identification and characterization of the genes encoding a unique surface (S-) layer of Tannerella forsythia. Gene. 2006;371:102-11.

6. Posch G, Sekot G, Friedrich V, Megson ZA, Koerdt A, Messner P, et al. Glycobiology aspects of the periodontal pathogen Tannerella forsythia. Biomolecules. 2012;2:467-82.

7. Sekot G, Schuster D, Messner P, Pum D, Peterlik H, Schäffer C. Small-angle Xray scattering for imaging of surface layers on intact bacteria in the native environment. J Bacteriol. 2013:195:2408-14.

8. Bloch S, Tomek MB, Friedrich V, Messner P, Schäffer C. Nonulosonic acids contribute to the pathogenicity of the oral bacterium Tannerella forsythia. Interface Focus. 2019;9:20180064.

9. Sekot G, Posch G, Messner P, Matejka M, Rausch-Fan X, Andrukhov O, et al. Potential of the Tannerella forsythia S-layer to delay the immune response. J Dent Res. 2011;90:109-14.

10. Tomek MB, Maresch D, Windwarder M, Friedrich V, Janesch B, Fuchs K, et al. A general protein O-glycosylation gene cluster encodes the species-specific glycan of the oral pathogen Tannerella forsythia: O-glycan biosynthesis and immunological implications. Front Microbiol. 2018;9:2008.

11. Coyne MJ, Fletcher CM, Chatzidaki-Livanis M, Posch G, Schaffer C, Comstock LE. Phylum-wide general protein O-glycosylation system of the Bacteroidetes. Mol Microbiol. 2013;88:772-83.

12. Tomek MB, Neumann L, Nimeth I, Koerdt A, Andesner P, Messner P, et al. The S-layer proteins of Tannerella forsythia are secreted via a type IX secretion system that is decoupled from protein O-glycosylation. Mol Oral Microbiol. 2014:29:307-20.

13. Lasica AM, Ksiazek M, Madej M, Potempa J. The type IX secretion system (T9SS): highlights and recent insights into its structure and function. Front Cell Infect Microbiol. 2017;7:215.

14. Megson ZA, Koerdt A, Schuster H, Ludwig R, Janesch B, Frey A, et al. Characterization of an a-l-fucosidase from the periodontal pathogen Tannerella forsythia. Virulence. 2015;6:282-92.

15. Roy S, Douglas CWI, Stafford GP. A novel sialic acid utilization and uptake system in the periodontal pathogen Tannerella forsythia. J Bacteriol. 2010; 192:2285-93.

16. Roy S, Phansopa C, Stafford P, Honma K, Douglas CWI, Sharma A, et al. Betahexosaminidase activity of the oral pathogen Tannerella forsythia influences biofilm formation on glycoprotein substrates. FEMS Immunol Med Microbiol. 2012;65:116-20.

17. Honma K, Ruscitto A, Frey AM, Stafford GP, Sharma A. Sialic acid transporter NanT participates in Tannerella forsythia biofilm formation and survival on epithelial cells. Microb Pathog. 2016;94:12-20.

18. Ksiazek M, Mizgalska D, Eick S, Thøgersen IB, Enghild JJ, Potempa J. KLIKK proteases of Tannerella forsythia: putative virulence factors with a unique domain structure. Front Microbiol. 2015:6:312.

19. Chen T, Abbey K, Deng W, Cheng M. The bioinformatics resource for oral pathogens. Nucleic Acids Res. 2005;33(Web Server issue):W734-40.

20. Friedrich $V$, Pabinger $S, C$ Chen $T$, Messner $P$, Dewhirst FE, Schäffer $C$. Draft genome sequence of Tannerella forsythia type strain ATCC 43037. Genome Announc. 2015;3:e00660-15.

21. Friedrich $V$, Janesch B, Windwarder M, Maresch D, Braun ML, Megson ZA, et al. Tannerella forsythia strains display different cell-surface nonulosonic acids: biosynthetic pathway characterization and first insight into biological implications. Glycobiology. 2017;27:342-57.

22. Endo A, Watanabe T, Ogata N, Nozawa T, Aikawa C, Arakawa S, et al. Comparative genome analysis and identification of competitive and cooperative interactions in a polymicrobial disease. ISME J. 2015;9: $629-42$.

23. Stafford GP, Chaudhuri RR, Haraszthy V, Friedrich V, Schäffer C, Ruscitto A, et al. Draft genome sequences of three clinical isolates of Tannerella forsythia isolated from subgingival plaque from periodontitis patients in the United States. Genome Announc. 2016:4:e01286-16.

24. Hanson-Drury S, To TT, Liu Q, Vo AT, Kim M, Watling M, et al. Draft genome sequence of Tannerella forsythia clinical isolate 9610. Genome Announc. 2017;5:e00024-17 
25. Haigh RD, Crawford LA, Ralph JD, Wanford JJ, Vartoukian SR, Hijazi K, et al. Draft whole-genome sequences of periodontal pathobionts Porphyromonas gingivalis, Prevotella intermedia, and Tannerella forsythia contain phase-variable restriction-modification systems. Genome Announc. 2017;5:e01229-17.

26. Züger J, Lüthi-Schaller H, Gmür R. Uncultivated Tannerella BU045 and BU063 are slim segmented filamentous rods of high prevalence but low abundance in inflammatory disease-associated dental plaques. Microbiology (Reading). 2007;153(Pt 11):3809-16

27. Vartoukian SR, Moazzez RV, Paster BJ, Dewhirst FE, Wade WG. First cultivation of health-associated Tannerella sp. HOT-286 (BU063). J Dent Res. 2016;95:1308-13.

28. Beall CJ, Campbell AG, Dayeh DM, Griffen AL, Podar M, Leys EJ. Single cell genomics of uncultured, health-associated Tannerella BU063 (Oral taxon 286) and comparison to the closely related pathogen Tannerella forsythia. PLoS One. 2014;9:e89398.

29. Beall CJ, Campbell AG, Griffen AL, Podar M, Leys EJ. Genomics of the Uncultivated, Periodontitis-Associated Bacterium Tannerella sp. BU045 (Oral Taxon 808). mSystems. 2018;3:e00018-18.

30. Ondov BD, Treangen TJ, Melsted P, Mallonee AB, Bergman NH, Koren S, et al. Mash: fast genome and metagenome distance estimation using MinHash. Genome Biol. 2016;17:132

31. Sharma A. Virulence mechanisms of Tannerella forsythia. Periodontol 2000. 2010;54:106-16.

32. Honma K, Mishima E, Sharma A. Role of Tannerella forsythia NanH sialidase in epithelial cell attachment. Infect Immun. 2011;79:393-401.

33. Yoo JY, Kim HC, Zhu W, Kim S-M, Sabet M, Handfield M, et al. Identification of Tannerella forsythia antigens specifically expressed in patients with periodontal disease. FEMS Microbiol Lett. 2007;275:344-52.

34. Sahl JW, Caporaso JG, Rasko DA, Keim P. The large-scale blast score ratio (LS-BSR) pipeline: a method to rapidly compare genetic content between bacterial genomes. PeerJ. 2014;2. https://doi.org/10.7717/peerj.332.

35. Medini D, Donati C, Tettelin H, Masignani V, Rappuoli R. The microbial pangenome. Curr Opin Genet Dev. 2005;15:589-94.

36. Tettelin H, Masignani V, Cieslewicz MJ, Donati C, Medini D, Ward NL, et al. Genome analysis of multiple pathogenic isolates of Streptococcus agalactiae: implications for the microbial "pan-genome". Proc Natl Acad Sci U S A. 2005; 102:13950-5.

37. Che D, Hasan MS, Chen B. Identifying pathogenicity islands in bacterial pathogenomics using computational approaches. Pathogens. 2014;3:36-56.

38. Martens EC, Koropatkin NM, Smith TJ, Gordon J. Complex glycan catabolism by the human gut microbiota: the Bacteroidetes Sus-like paradigm. J Biol Chem. 2009;284:24673-7.

39. Grondin JM, Tamura K, Déjean G, Abbott DW, Brumer H. Polysaccharide Utilization Loci: Fueling Microbial Communities. J Bacteriol. 2017;199: e00860-16.

40. Schäffer C, Messner P. Emerging facets of prokaryotic glycosylation. FEMS Microbiol Rev. 2017:41:49-91.

41. Reeves PR, Cunneen MM, Liu B, Wang L. Genetics and evolution of the Salmonella galactose-initiated set of o antigens. PLoS One. 2013;8:e69306.

42. Ikemura T. Codon usage and tRNA content in unicellular and multicellular organisms. Mol Biol Evol. 1985;2:13-34.

43. Kanaya S, Yamada Y, Kudo Y, Ikemura T. Studies of codon usage and tRNA genes of 18 unicellular organisms and quantification of Bacillus subtilis tRNAs: gene expression level and species-specific diversity of codon usage based on multivariate analysis. Gene. 1999;238:143-55.

44. Carbone A, Zinovyev A, Képès F. Codon adaptation index as a measure of dominating codon bias. Bioinformatics. 2003;19:2005-15.

45. O'Neill PK, Or M, Erill I. scnRCA: a novel method to detect consistent patterns of translational selection in mutationally-biased genomes. Plos One. 2013;8:e76177.

46. Carbone A, Képès F, Zinovyev A. Codon bias signatures, organization of microorganisms in codon space, and lifestyle. Mol Biol Evol. 2005;22:547-61.

47. Novick RP, Ram G. The floating (pathogenicity) island: a genomic dessert. Trends Genet. 2016:32:114-26.

48. Bolger AM, Lohse M, Usadel B. Trimmomatic: a flexible trimmer for Illumina sequence data. Bioinformatics. 2014;30:2114-20.

49. Luo R, Liu B, Xie Y, Li Z, Huang W, Yuan J, et al. SOAPdenovo2: an empirically improved memory-efficient short-read de novo assembler. Gigascience. 2012;1:18

50. Gurevich A, Saveliev V, Vyahhi N, Tesler G. QUAST: quality assessment tool for genome assemblies. Bioinformatics. 2013;29:1072-5.
51. Robinson JT, Thorvaldsdóttir H, Winckler W, Guttman M, Lander ES, Getz G, et al. Integrative genomics viewer. Nat Biotechnol. 2011;29:24-6.

52. Thorvaldsdóttir H, Robinson JT, Mesirov JP. Integrative genomics viewer (IGV): high-performance genomics data visualization and exploration. Brief Bioinformatics. 2013;14:178-92.

53. Felsenstein J. PHYLIP (Phylogeny Inference Package) version 3.6. Distributed by the author. Seattle: Department of Genome Sciences, University of Washington; 2005. http://evolution.genetics.washington.edu/phylip.html. Accessed 15 Feb 2019

54. Junier T, Zdobnov EM. The Newick utilities: high-throughput phylogenetic tree processing in the UNIX shell. Bioinformatics. 2010;26:1669-70.

55. Darling AE, Mau B, Perna NT. progressiveMauve: multiple genome alignment with gene gain, loss and rearrangement. PLoS One. 2010;5:e11147.

56. Altschul SF, Madden TL, Schäffer AA, Zhang J, Zhang Z, Miller W, et al. Gapped BLAST and PSI-BLAST: a new generation of protein database search programs. Nucleic Acids Res. 1997;25:3389-402.

57. Camacho C, Coulouris G, Avagyan V, Ma N, Papadopoulos J, Bealer K, et al. BLAST+: architecture and applications. BMC Bioinformatics. 2009; $10: 421$.

58. Kurtz S, Phillippy A, Delcher AL, Smoot M, Shumway M, Antonescu C, et al. Versatile and open software for comparing large genomes. Genome Biol. 2004;5:R12.

59. Contreras-Moreira B, Vinuesa P. GET_HOMOLOGUES, a versatile software package for scalable and robust microbial pangenome analysis. Appl Environ Microbiol. 2013;79:7696-701.

60. Li L, Stoeckert CJ, Roos DS. OrthoMCL: identification of ortholog groups for eukaryotic genomes. Genome Res. 2003;13:2178-89.

61. Kristensen DM, Kannan L, Coleman MK, Wolf YI, Sorokin A, Koonin EV, et al. A low-polynomial algorithm for assembling clusters of orthologous groups from intergenomic symmetric best matches. Bioinformatics. 2010;26:1481-7.

62. Yin $Y$, Mao $X$, Yang J, Chen $X$, Mao F, Xu Y. dbCAN: a web resource for automated carbohydrate-active enzyme annotation. Nucleic Acids Res. 2012 40(Web Server issue):W445-51.

63. Marchler-Bauer A, Bo Y, Han L, He J, Lanczycki CJ, Lu S, et al. CDD/SPARCLE: functional classification of proteins via subfamily domain architectures. Nucleic Acids Res. 2017:45:D200-3.

64. Marchler-Bauer A, Derbyshire MK, Gonzales NR, Lu S, Chitsaz F, Geer LY, et al. CDD: NCBI's conserved domain database. Nucleic Acids Res. 2015; 43(Database issue):D222-6.

65. Marchler-Bauer A, Lu S, Anderson JB, Chitsaz F, Derbyshire MK, DeWeeseScott C, et al. CDD: a conserved domain database for the functional annotation of proteins. Nucleic Acids Res. 2011;39(Database issue):D225-9.

66. Marchler-Bauer A, Bryant SH. CD-Search: protein domain annotations on the fly. Nucleic Acids Res. 2004;32(Web Server issue):W327-31.

67. Winter S, Jahn K, Wehner S, Kuchenbecker L, Marz M, Stoye J, et al. Finding approximate gene clusters with gecko 3. Nucleic Acids Res. 2016;44:9600-10.

68. Wittkop T, Emig D, Lange S, Rahmann S, Albrecht M, Morris JH, et al. Partitioning biological data with transitivity clustering. Nat Methods. 2010:7:419-20.

69. Wright F. The "effective number of codons" used in a gene. Gene. 1990:87:23-9.

\section{Publisher's Note}

Springer Nature remains neutral with regard to jurisdictional claims in published maps and institutional affiliations.

Ready to submit your research? Choose BMC and benefit from:

- fast, convenient online submission

- thorough peer review by experienced researchers in your field

- rapid publication on acceptance

- support for research data, including large and complex data types

- gold Open Access which fosters wider collaboration and increased citations

- maximum visibility for your research: over $100 \mathrm{M}$ website views per year

At BMC, research is always in progress.

Learn more biomedcentral.com/submissions 